Análisis de los incentivos económicos en la capacidad instalada de energía solar fotovoltaica en Colombia

Manuela Castaño-Gómez y John Jairo García-Rendón 
Lecturas de Economía, 93 (julio-diciembre 2020), pp. 23-64

Manuela Castaño-Gómezy John Jairo García-Rendón

\section{Análisis de los incentivos económicos en la capacidad instalada de energía solar fotovoltaica en Colombia}

Resumen: Debido a la caída abrupta a nivel mundial del costo nivelado de las tecnologías renovables no convencionales para la generación de energía con estas fuentes (en el caso de los mercados en Estados Unidos entre 39 y 152 USD/MWh para la energía solar fotovoltaica y entre 26 y 50 USD/MWh para la eólica), se ha sustituido la utilización de mecanismos como las Feed in Tariff por las subastas, como un mecanismo de mercado viable para incentivar la inversión en estas fuentes de generación. Por medio del modelo de difusión tecnológica de Bass, este artículo analiza el impacto que tienen los incentivos actuales, contemplados en la Ley 1715 de 2014, en la inversión en energía solar fotovoltaica en Colombia. Los principales resultados arrojados por el escenario de un proyecto a gran escala muestran una capacidad instalada de energía solar fotovoltaica de $1.542 \mathrm{MW}$ en 2030, lo que lo hace el más rentable y el de mayor potencial de crecimiento. El aporte del articulo es examinar el potencial que tiene el mercado eléctrico colombiano respecto a la inclusión de energía solar fotovoltaica.

Palabras clave: gestión de la energía; regulación económica; fuentes no convencionales de energía renovables; innovación tecnológica.

Clasificación JEL: K2, L21, L 51.

\section{Installed capacity of photovoltaic solar energy in Colombia: An analysis of economic incentives}

Abstract: Due to the abrupt drop in the worldwide energy generation levelized cost of non-conventional renewable technologies (in the case of the markets in the United States between 39 to 152 USD/MWh forphotovoltaic solar energy and between 26 to 50 USD/MWh for wind energy), the use of mechanisms such as Feed in Tariff have been replaced by auctions, as a viable market mechanism to encourage investment on these generation sources. Based on Bass's technology diffusion model, this paper analyzes the impact of current incentives, considered in Law 1715 of 2014, on investment in photovoltaic solar energy in Colombia. The main results of the large-scale project scenario show an installed capacity 1542 MW of solar PV by 2030, which makes it the most profitable and the one with the highest growth potential. The contribution in this paper is to survey the Colombian electricity market potential on the inclusion of solar PV.

Keywords: energy management; economic regulation; non-conventional renewable energy; technological innovation.

https://doi.org/10.17533/udea.le.n93a338727

\section{(cC) BY-NC-SA}

Este artículo y sus anexos se distribuyen por la revista Lecturas de Economía bajo los términos de la Licencia Creative Commons Atribución-NoComercial-CompartirIgual 4.0. https://creativecommons.org/licenses/by-nc-sa/4.0/ 


\section{Analyse des incitations économiques dans la capacité installée de l'énergie solaire photovoltaïque en Colombie}

Résumé: En raison de la chute brutale au niveau mondial de la production d'énergie à des tecbnologies renowvelables non conventionnelles (aux États-Unis une chute entre 39 et 152 USD/MWh pour l'énergie solaire photovoltaïque entre 26 et 50 USD/MWh pour l'énergie éolienne), l'utilisation du Feed in Tariff a été remplacée par des enchères, en tant que mécanisme de marché viable à fin d'encourager l'investissement dans ces sources d'énergie. Tout en utilisant le modèle de diffusion technologique de Bass, cet article analyse l'impact des incitations économiques de la loi 1715 de 2014, sur l'investissement dans la production d'énergie solaire photovoltaïque en Colombie. Les principaux résultats montrent qu'un projet à grande échelle aurait une capacité installée de $1542 \mathrm{MW}$ d'énergie solaire photovoltaïque pour l'année 2030. Il s'agit du projet le plus rentable et celui avec le plus grand potentiel de croissance. La contribution de l'article est d'examiner le potentiel du marché colombien de l'électricité en ce qui concerne l'inclusion de l'énergie solaire photovoltaïque.

Mots clés: gestion de l'énergie; réglementation économique; énergies renouvelables non conventionnelles; innovation technologique.

\section{Cómo citar / How to cite this item:}

Castaño-Gómez, M. \& García-Rendón, J. J. (2020). Análisis de los incentivos económicos en la capacidad instalada de energía solar fotovoltaica en Colombia. Lecturas de Economía, 93, 23-64.

https://doi.org/10.17533/udea.le.n93a338727 


\title{
Análisis de los incentivos económicos en la capacidad instalada de energía solar fotovoltaica en Colombia
}

\author{
Manuela Castaño-Gómez $\mathbb{1}^{\mathrm{a}}$ y John Jairo García-Rendón (ib ${ }^{\mathrm{b}}$ \\ -Introducción. -I. Marco teórico y revisión de literatura. -II. Metodología. -III. Resultados. \\ -Conclusiones. -Agradecimientos. -Referencias.
}

Primera versión recibida el 6 de junio de 2019; versión final aceptada el 8 de enero de 2020

\section{Introducción}

En la actualidad se percibe un cambio disruptivo en los mercados eléctricos a nivel mundial, en los cuales las redes inteligentes, incluida la infraestructura en medición avanzada, la generación distribuida, la automatización de la red y el almacenamiento, desempeñarán un papel fundamental en el funcionamiento de los mercados eléctricos. Además, la respuesta de la demanda y el prosumidor serán fundamentales en la prestación del servicio, para garantizar la confiabilidad del sistema, el uso eficiente de los recursos y la sostenibilidad económica, ambiental y social. Pero al mismo tiempo, los diseños de mecanismos y la fijación de principios regulatorios claros serán necesarios para garantizar una buena calidad en la prestación del servicio, en el que, seguramente, la dicotomía entre "liberalización" y "regulación" perderá sentido para explicar el funcionamiento de estos mercados.

El crecimiento de los recursos de generación distribuida empieza a jugar un rol fundamental para la prestación de los servicios de electricidad y ha cuestionado la organización clásica de los sistemas de energía para garantizar

a Manuela Castaño-Gómez: candidata a Magister en Economía Aplicada, Universidad EAFIT, Colombia. Dirección postal: A. A. 3300. Correo electrónico: mpcastanog@eafit.edu.co https:/ / orcid.org/0000-0002-5436-7903

b John Jairo García-Rendón: director del Grupo de Estudios en Economía y Empresa de la Universidad EAFIT, Colombia. Dirección postal: A. A. 3300. Medellín, Colombia. Correo electrónico: jgarcia@eafit.edu.co http://orcid.org/0000-0002-1269-2548 
la eficiencia energética. Dichos sistemas se basan en sistemas centralizados y plantea grandes interrogantes a nivel mundial sobre el carácter de los sistemas de energía futuros, en los que las fuentes no convencionales de energía renovables (FNCER) cada vez tienen mayor participación en generación de energía, máxime si se consideran los problemas ambientales, el calentamiento global y el compromiso adquirido en el Acuerdo de París en 2016 (Pillot et al., 2019). Como lo establecen Black y Veatch (2015), se espera que para el 2040 las fuentes de energía renovables tengan una participación del $40 \%$ de la capacidad total mundial; adicionalmente han sido consideradas como la cuarta revolución industrial en el sector energético.

Diferentes mecanismos se han implementado a nivel mundial para incentivar la inversión en fuentes de energía renovables. Contratos por diferencias, cuotas y subvenciones como las tarifas garantizadas fueron algunos de los mecanismos utilizados para garantizar a los generadores la recuperación de la inversión, pero con la disminución de los costos nivelados de estas fuentes, en la actualidad, ha sido posible utilizar mecanismos de mercado más eficientes como las subastas.

En 2019, en Colombia, la generación a partir de FNCER, específicamente la energía solar fotovoltaica (FV), es prácticamente nula aun cuando existe un gran potencial de generación a partir de este recurso, dada la localización geográfica que tiene nuestro país; además, esta fuente tiene una gran complementariedad con el recurso hidráulico, el cual conforma la mayor fuente de generación de electricidad en el mercado mayorista colombiano, puesto que, cuando se presenta El Niño, escasea el recurso hídrico pero aumenta la posibilidad de generar energía solar. Adicionalmente, la generación con este tipo de fuente puede hacerse por medio de la generación distribuida en el Sistema Interconectado Nacional (SIN), lo que conllevaría a que el consumidor tenga un papel activo, o a través de autogeneración en zonas no interconectadas (ZNI) y, así, los consumidores pueden tener acceso al servicio de electricidad en las regiones donde no es posible tener redes de transmisión y distribución. Esto hace importante analizar el impacto de los incentivos económicos en el comportamiento de la capacidad instalada de energía solar FV en Colombia, por tanto, este es el objetivo que persigue este estudio. Para realizar este análisis empezamos por revisar la experiencia de 
algunos mercados sobre el tema a nivel mundial, como en Alemania, China y Estados Unidos, y en Latinoamérica en países como Brasil, Chile y México.

En Colombia se empiezan a realizar cambios regulatorios importantes que propician la inclusión de FNCER y redes inteligentes. En 2014 se promulgó la Ley 1715, la cual establece los lineamientos de política para la promoción de las inversiones con FNCER. En 2018, por medio de la Resolución 40072 del Ministerio de Minas y Energía (MME) empiezan a definirse aspectos regulatorios para la implementación de la infraestructura en medición avanzada (AMI, por sus siglas en inglés) en el SIN. Las resoluciones 024 de 2015 y 030 de 2018 de la Comisión de Regulación de Energía y Gas (CREG) definen la regulación de las actividades de generación distribuida y la autogeneración a pequeña y gran escala en el SIN, y la resolución CREG 038 de 2018 estable las reglas para la actividad de autogeneración para zonas no interconectas. Además, con la Resolución 098 de 2019, de la CREG se da un paso importante en términos de almacenamiento, ya que esta define las condiciones para la incorporación de sistemas de almacenamiento con baterías en el SIN.

También por medio de las resoluciones 40791 y 40795 del MME se realizó la convocatoria, en febrero de 2019, para la primera subasta de contratos de largo plazo de energía eléctrica (a 10 años), la cual buscaba incentivar la instalación de FNCER, pero desafortunadamente no se adjudicó. No obstante, en la segunda subasta para contratos de largo plazo con FNCER, realizada en octubre de 2019, por medio de la Resolución 40590 de 2019, se adjudicaron 1298 MW de capacidad instalada, 5 proyectos de energía eólica y 3 solares. Por su parte, en marzo de 2019, se llevó a cabo una subasta de energía firme para el cargo por confiabilidad y, si bien el objeto no eran las FNCER, se terminaron adjudicando 1398 MW con estas fuentes, 6 proyectos eólicos y 2 solares, para instalarse en 2022.

En este estudio utilizamos el modelo de difusión tecnológica de Bass para analizar el efecto que tienen los incentivos actuales, contemplados en la Ley 1715 de 2014, sobre la inversión en energía solar FV en Colombia. Si bien se han utilizado modelos de agentes y de paridad tarifaria, este modelo ha sido uno de los más utilizados para pronosticar la difusión y demanda de tecnología, ya que con poca información permite segmentar los agentes de acuerdo al periodo 
Castaño-Gómez y García-Rendón: Análisis de los incentivos económicos en la capacidad...

en que se adopta un nuevo producto, además incluye coeficientes de imitación e innovación, que permiten identificar el número de adoptantes de una tecnología (Bass, 2004). En cambio, aunque el modelo basado en agentes permite simular las interacciones estratégicas de los individuos en una población, presenta una gran limitación para su implementación a gran escala, debido a la cantidad de datos requerida (Palmer et al., 2015). Por su parte, el modelo de paridad tarifaria, el cual se da cuando la tarifa de la energía generada con una fuente —en este caso la solar fotovoltaica - es menor que la cobrada por el comercializador, pero es importante aclarar que el hecho de que una tecnología sea más barata que otra no implica que necesariamente será implementada en un mercado (Konzen \& Zilles, 2014).

Los principales resultados arrojados muestran que un escenario de un proyecto a gran escala, el cual considera una capacidad instalada de 5,8 MW e incorpora los incentivos establecidos por la Ley 1715 de 2014, es el más rentable y el de mayor potencial de crecimiento. Este arroja un potencial de una capacidad instalada de 1542 MW en 2030, un poco más si se compara con los pronósticos del Plan de Expansión de la Unidad de Planeación Minero Energética (UPME, 2017) en el que se plantean 1090 MW instalados para este mismo año.

El presente trabajo comprende las siguientes secciones: después de esta introducción se presenta el marco teórico y la revisión de literatura en la sección I. La sección II contiene los aspectos metodológicos utilizados para la elaboración de los escenarios. La sección III presenta los resultados y, finalmente se dan a conocer las conclusiones.

\section{Revisión de literatura}

Como lo establecen Laffont y Tirole (1993), los incentivos en una economía se convierten en uno de los principales determinantes de la conducta de los agentes. Diversos autores analizan el impacto de los incentivos regulatorios en el crecimiento de la tecnología solar FV. Por ejemplo, Mints (2011) identifica las oportunidades y consecuencias que han generado estos incentivos en las diferentes regiones. Análogamente, do Santos y Cunha (2019) y Washburn y Pablo-Romero (2019) estudian el caso de los incentivos regulatorios en América Latina, examinando cómo ha sido la experiencia de las políticas energéticas 
para los países de la región. Asimismo, los incentivos fiscales también han desempeñado un papel importante para una mayor inversión y utilización de FNCER a nivel mundial (Hille et al., 2020; Zhao et al., 2016).

Como lo establecen Bugrahan et al. (2011), la regulación ha tenido un papel trascendente para la expansión de esta tecnología, tanto o más que la disponibilidad de recursos naturales. Entre los principales incentivos que se han implementado para contrarrestar los altos costos y las barreras regulatorias para aumentar el uso de FNCER, se destacan las tarifas garantizadas, los incentivos fiscales, los instrumentos para la reducción de emisiones de $\mathrm{CO}_{2}$, las cuotas y los contratos por diferencias entre otros.

- Tarifas garantizas: quizás este es uno de los incentivos más utilizados para la inclusión de FNCER. Como su nombre lo indica, consiste en garantizar o fijar un precio de compra para los generadores de energía renovable, fijados a partir del costo de la producción de este tipo de energía (Winter \& Schlesewsky, 2019; Böhringer et al., 2017).

- Incentivos fiscales: los más comunes han sido las exenciones de IVA y aranceles, deducción en el impuesto a la renta, depreciación acelerada, créditos fiscales, subsidios, préstamos o inversiones directas del estado (Hille et al., 2020; Moreno, 2017; Zhao et al., 2016; UPME, 2015a).

- Instrumentos para la reducción de emisiones: como el impuesto y topes de emisiones de $\mathrm{CO}_{2}$ (Boyce, 2018).

- Cuotas: se basa en fijar porcentajes específicos para la matriz energética de un país, lo que permite diversificarla poniendo metas fijas. En este tipo de mecanismos se encuentran las subastas (estos dan como resultado contratos de energía en firme, otorgados a través de licitaciones públicas) y los Certificados de Energía Renovable (se otorgan certificados a los generados de ER que pueden ser comercializados, generalmente representan la producción de un MWh de generación de energía limpia) (UPME, 2015a).

- Contratos por diferencias: es un incentivo directo que garantiza a los productores de ER un precio de electricidad competitivo al mercado mayorista. De manera que los generadores reciben una prima de 
Castaño-Gómez y García-Rendón: Análisis de los incentivos económicos en la capacidad...

la diferencia entre el precio del ejercicio y el precio del mercado (UPME, 2015a).

Los casos de éxito de expansión de esta tecnología se caracterizan por tener altas inversiones, las cuales han tenido un crecimiento significativo a nivel mundial. En la Figura 1 se puede identificar su comportamiento entre 2004 y 2017. Estas inversiones tienen diferentes fuentes, la más representativa ha sido la financiación de activos, ya sea bajo el modelo de negocio de arrendamiento, de bonos o de otras opciones de la banca; en este sentido ha sido fundamental que la regulación fomente la participación de las entidades financieras en los proyectos solares FV.

Figura 1. Mecanismos de financiación en proyectos solares FV

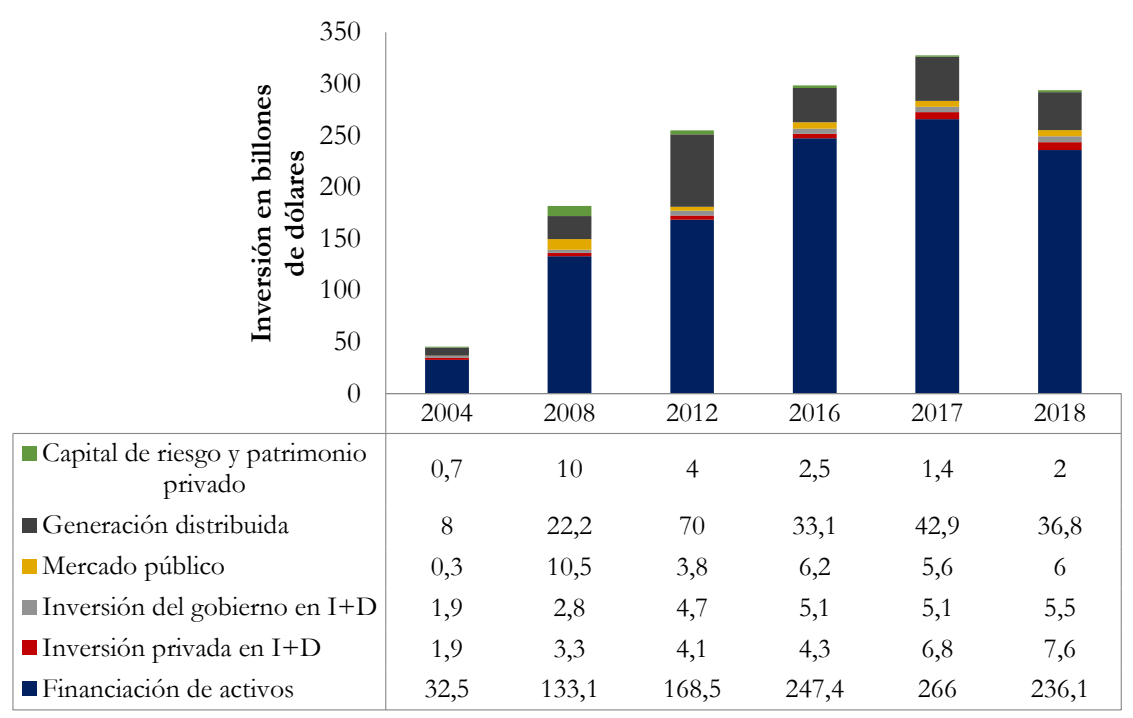

Fuente: elaboración propia.

\section{A. Energía renovable en el mundo}

A finales de 2017 a nivel mundial había una capacidad instalada de energías renovables de 2179,43 GW (IRENA, 2019), de la cual, la mitad proviene de 
fuentes hídricas, seguido de la energía eólica y solar, como se muestra en la Figura 2.

Figura 2. Participación de fuentes renovables en la capacidad instalada a nivel mundial -Hidraúlica Marina Eólica $\square$ Solar $\backsim$ Biomasa Geotérmica

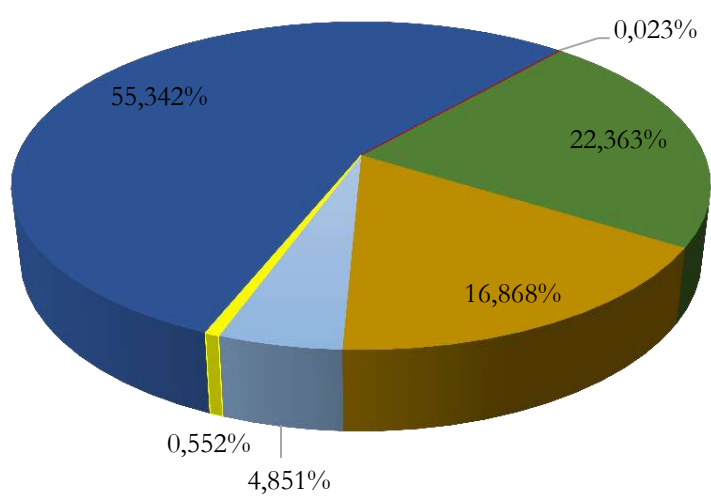

Fuente: elaboración propia a partir de (IRENA, 2019).

La capacidad instalada de energía solar FV a finales de 2017 alcanzó los 384,62 GW, más del 30\% del 2016, lo que representa en energía generada 317,67 GWh (IRENA, 2019). En cuanto al potencial de crecimiento de esta tecnología, proyecciones como las de Bloomberg New Energy Finance (Bullard, 2018) señalan que para el año 2040 el $60 \%$ de la capacidad mundial de energía provendrá de fuentes de energía no contaminantes, de las cuales las tecnologías eólica y solar se convertirán en las formas más baratas de producir electricidad en muchos países durante la década de 2020 y en la mayor parte del mundo en la década de 2030. La Tabla 1 muestra la capacidad solar FV acumulada y la proyección de la misma a 2040.

Tabla 1. Capacidad solar FV acumulada y proyección a 2040 a nivel mundial

\begin{tabular}{lcccccc}
\hline Capacidad eléctrica/Año & $\mathbf{2 0 0 9}$ & $\mathbf{2 0 1 1}$ & $\mathbf{2 0 1 5}$ & $\mathbf{2 0 1 7}$ & $\mathbf{2 0 2 0}$ & $\mathbf{2 0 4 0}$ \\
\hline Capacidad solar FV [GW] & 22,48 & 69,59 & 220,28 & 384,62 & 500,00 & 2000,00 \\
\hline
\end{tabular}

Fuente: elaboración propia a partir de (IRENA, 2019). 
Castaño-Gómez y García-Rendón: Análisis de los incentivos económicos en la capacidad...

Asimismo, el costo nivelado de la generación de energía (LCOE, por sus siglas en inglés), es una medida del costo neto actual promedio de la generación de electricidad para una planta generadora a lo largo de su vida útil $y$, por tanto, consiste en el precio que debe cobrarse por la energía generada durante la vida útil de la planta, de tal manera que sea posible recuperar los costos de la inversión. Específicamente en el caso de la energía FV, el costo de generación se ha reducido considerablemente en la última década, como se observa en la Figura 3. Además, para el año 2040 se proyectan reducciones de estos costos del orden del $60 \%$, esto debido al desarrollo tecnológico que ha traído la curva de aprendizaje de esta tecnología, lo que permite la implementación de economías de escala. Esto es coherente con la Ley de Swanson, la cual indica que, con cada duplicación de la producción de paneles solares, la reducción de sus costos ha sido del 20\%. La tipología más común son los paneles de silicio cristalino; estos son los líderes del mercado con una participación a 2016 del 85\% (IRENA, 2018b; Hunt, 2014).

Figura 3. Capacidad instalada FV vs. Costo nivelado de la energía solar FV

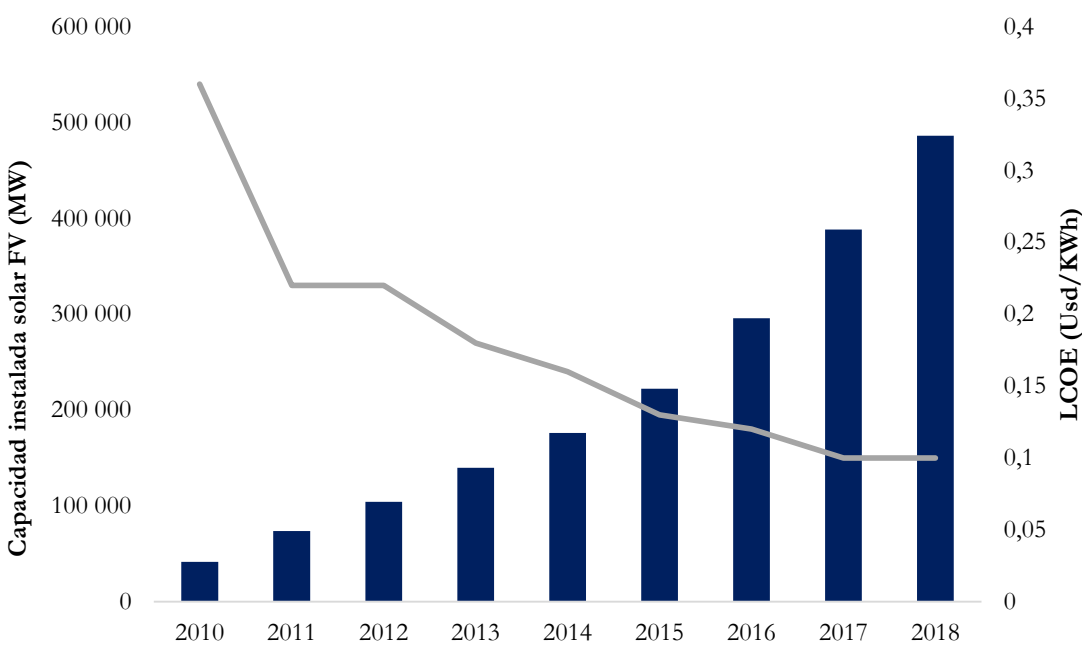

Fuente: elaboración propia a partir de (IRENA, 2019).

Los líderes mundiales en generación solar FV son: China, con una capacidad instalada a 2017 de 130,4 GW; Estados Unidos, con 85,3 GW; 
Japón, con 63,3 GW; India, que cuenta con 57,4 GW y, por su parte, Alemania cuenta con 48,4 GW (IRENA, 2019).

Como puede observarse, China es potencia en esta materia, resultado de combinar una ubicación geográfica privilegiada en el cinturón solar y las diferentes políticas que apuntan al alto desarrollo de este sector. Por otro lado, Alemania es uno de los pioneros en la creación de incentivos para la masificación de energías renovables, lo cual le ha permitido ser potencia a pesar de no contar con los mejores niveles de radiación solar. En la Figura 4 puede observarse mejor la expansión en la capacidad solar FV instalada en estas economías.

Figura 4. Evolución de la capacidad solar FV en Alemania, China y EE. UU.

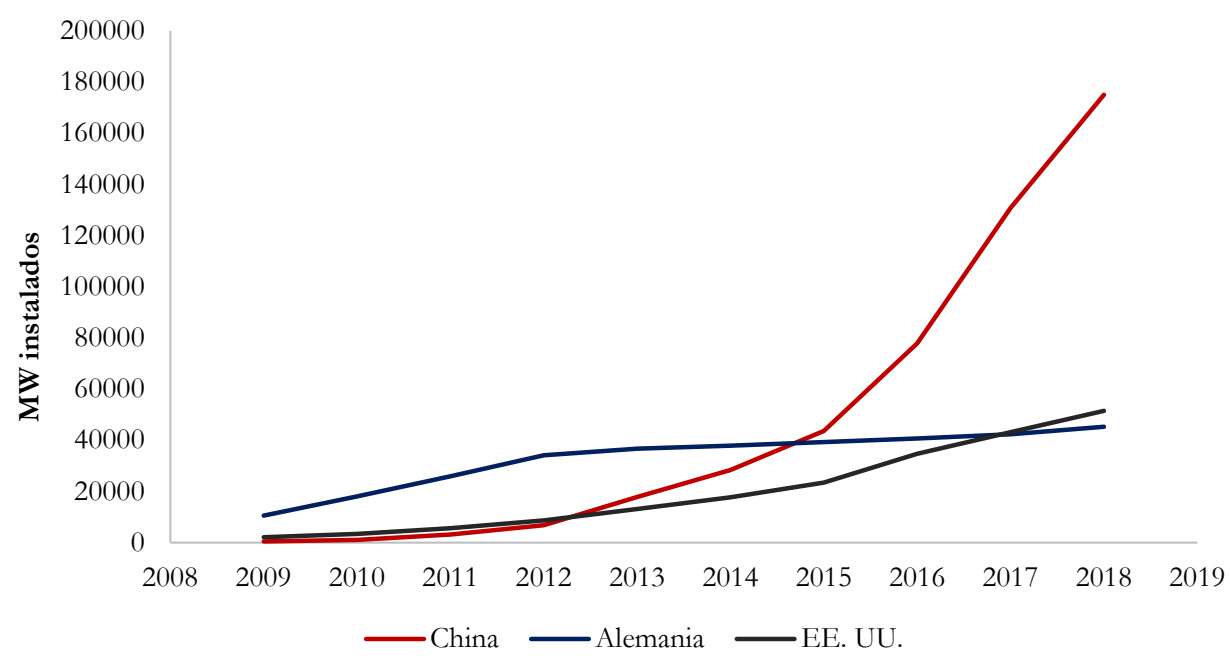

Fuente: elaboración propia a partir de (IRENA, 2019).

Para el año 2000, Alemania era pionera en el esquema de aranceles y programas de techos solares; además, contaba con una prima que se les otorgaba a las empresas de servicios públicos por integrar la energía solar a la red e incentivos por medio de las tarifas garantizadas. Como resultado se dio un gran estímulo a la demanda de esta fuente de energía en el país (Mints, 2011). Por otra parte, Estados Unidos ha implementado programas de cartera 
Castaño-Gómez y García-Rendón: Análisis de los incentivos económicos en la capacidad...

renovable —utilizando también las tarifas garantizadas — los cuales impactan directamente la tasa de retorno de estos proyectos.

En resumen, estos países han logrado esta posición gracias a diferentes aspectos, como su nivel de radiación, nivel de industrialización, incentivos regulatorios con altas subvenciones, inversión público-privada, subsidios y programas de remuneración de medición neta y FIT (Femin et al., 2016).

\section{B. Energía renovable en Latinoamérica y El Caribe}

Por su parte, en Latinoamérica y el Caribe se espera un crecimiento en energía renovable de 1,4 GW en el 2015 a 10 GW en 2021 (UPME, 2015a). En la actualidad, las energías renovables con mayor potencial en América Latina son la solar FV, la eólica y la biomasa, debido a su ubicación geográfica y sus condiciones atmosféricas. Adicionalmente, los países de la región han hecho diferentes esfuerzos a nivel regulatorio con el fin de incentivar estas fuentes de energía: algunos de ellos han realizado diversos esfuerzos para promover las FNCER, y más del $80 \%$ han establecido objetivos estratégicos a largo plazo para lograrlo. La normativa más común para lograr estas metas son incentivos tributarios y las subastas (Washburn \& Pablo-Romero, 2019).

Los líderes a nivel de Latinoamérica son Brasil, Chile y México, los cuales atribuyen su éxito a la inserción de políticas que incentivan la inversión tanto pública como privada, además de las condiciones privilegiadas a nivel geográfico, especialmente en el caso de México, que cuenta con uno de los niveles más altos de radiación de la región. La Figura 5 presenta la tendencia de crecimiento de estos países contrastada con el caso colombiano, el cual, aunque se han hecho algunos esfuerzos recientes, está más atrasado comparativamente con otros países de la región.

A continuación, se describen los mercados de Chile, Brasil y México, que, como se ha dicho, son los más avanzados de la región.

\section{Chile}

Junto con Panamá, México y Nicaragua, Chile es el país con mayor número de estrategias, leyes o programas que afectan la producción de energía solar FV. En 2015 este país fue uno de los que más invirtió en esta temática 
a nivel mundial, y para el año 2050 tiene prevista la inclusión del $70 \%$ de su matriz energética con este tipo de fuentes (Simsek et al., 2019). Para lograrlo la Comisión Nacional de Energía propuso un esquema regulatorio de 12 políticas que incluye incentivos para la autogeneración, generación distribuida y cogeneración de energía, así como un esquema de subastas y tarifas horarias. Como respuesta, en 2017 la energía solar tuvo una participación del 7\%, con una capacidad instalada de 2,11 MW, mientras que en el 2016 solo tuvo $1 \%$ de participación. Además, se tienen perspectivas de crecimiento mayores, ya que el $67 \%$ de los proyectos de generación eléctrica en el país son de origen solar fotovoltaico (IRENA, 2019).

Figura 5. Evolución de la capacidad solar FV en algunos países de Latinoamérica

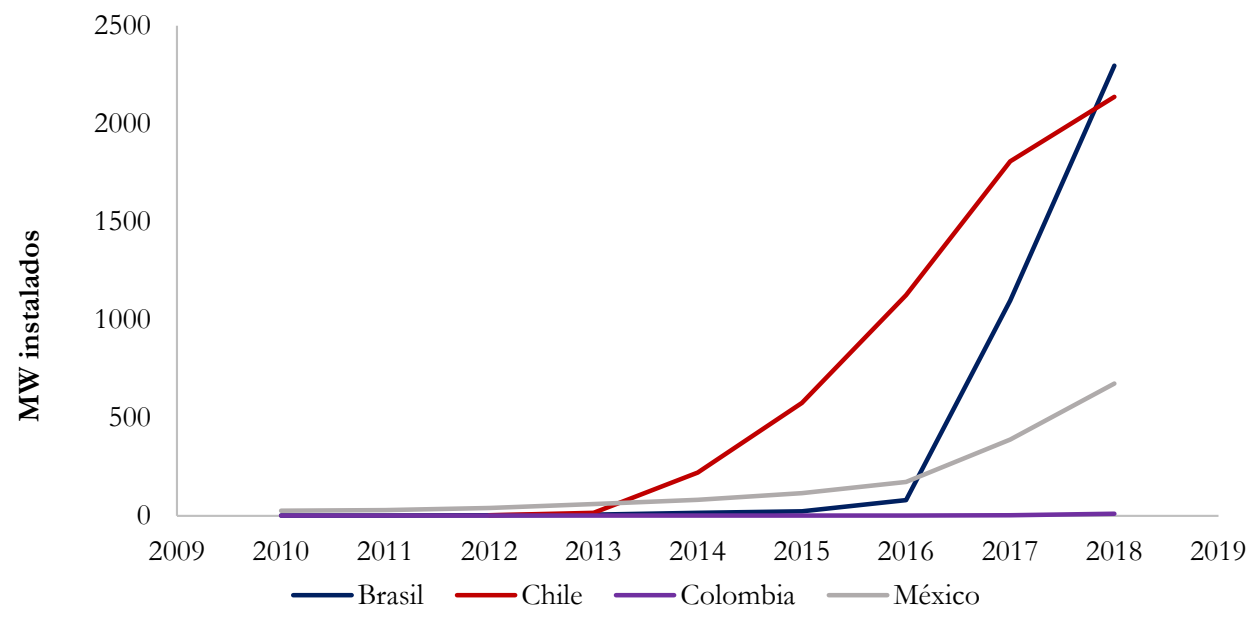

Fuente: elaboración propia a partir de (IRENA, 2019).

Desde 2014, lo usuarios conectados a la red han podido vender los excedentes de cogeneración si su capacidad no supera los $100 \mathrm{~kW}$. Adicionalmente, los proyectos de ER poseen una exención del 100\% de los impuestos sobre la transmisión si generan menos de 9 MW, y tienen una exención parcial si generan entre 9 y 20 MW (Saldivia et al., 2019). En Chile desincentivan el uso de combustibles fósiles mediante un impuesto al carbón que consiste en USD $\$ 5$ a las emisiones de $\mathrm{CO}_{2}$, aplicada a la generación térmica con una entrada de $50 \mathrm{MW}$, exceptuando la biomasa. Finalmente, han 
Castaño-Gómez y García-Rendón: Análisis de los incentivos económicos en la capacidad...

realizado un importante número de subastas en energías renovables, lo cual ha permitido que se haya reducido el precio de la electricidad (Washburn \& Pablo-Romero, 2019).

\section{Brasil}

Brasil, igual que Colombia, depende en gran medida del agua para generar energía, lo que implica deforestación y emisión de gases de efecto invernadero. Por esta razón, en años recientes se han hecho esfuerzos para fomentar el crecimiento de la generación de energía con FNCER, lo que ha resultado en un proceso de transición energética, soportado principalmente en iniciativas gubernamentales (do Santos \& Cunha, 2019). A través de La Agencia Nacional de Energía Eléctrica (ANEEL) se han formulado e implementado 17 políticas públicas con el fin de incentivar la inversión en energías limpias. Así, en 2015 se posicionó como el octavo país a nivel mundial con generación eólica, y en 2017 se construyó la central más grande de energía solar FV, la cual tiene una capacidad instalada de 1097 MW y suministra energía a 420000 hogares brasileños (IRENA, 2019).

Entre los incentivos adoptados por este país para implementar una estrategia de energía renovable se encuentran las exenciones de impuestos y las tarifas garantizadas, este último mecanismo fue adoptado en 2004, pero reemplazado en 2011 por subastas de dos tipos: generales y para tecnologías específicas. En total se han realizado 31 subastas, de las cuales 14 han sido para tecnologías específicas y 2 para energía solar FV. Cabe anotar que se han otorgado subsidios sobre todo en los costos de inversión de plantas de generación en zonas no interconectadas. Adicionalmente, desde 2012 se han realizado esfuerzos en materia de generación distribuida a través de un esquema de medición neta (Washburn \& Pablo-Romero, 2019).

\section{México}

Por su ubicación geográfica y nivel de radiación, México es uno de los cinco países a nivel mundial con mayor potencial para generar energía solar FV. Gracias a esto ha aumentado considerablemente su capacidad instalada, y para el año 2017 generar 570 GW (IRENA, 2018). 
Desde 2013 este país ha venido trabajando en 39 políticas para incentivar las energías no convencionales, de esta regulación se desprendió la reforma energética, la cual ha traído oportunidades para la diversificación de la matriz energética del país Azteca, por lo que, para 2050, se espera un 50\% de participación de energías limpias y eliminar diferentes barreras que presenta la implementación de este tipo de generación (IRENA, 2019). En México, los lineamientos de política en generación distribuida fueron dictados por la Ley de la Industria Eléctrica — publicada en 2014 — con el objetivo de promover el desarrollo sustentable de la industria eléctrica, de garantizar su operación continua, eficiente y confiable y de reducir las emisiones contaminantes. De otro lado, la Ley de Transición Energética — publicada en 2015- se enfoca en regular el uso óptimo de las fuentes no convencionales de energía, velando por la competitividad del país (Ley de transición energética México, 2015).

Desde 1982 se creó un fondo de investigación en energía sostenible, lo que ha permitido grandes desarrollos en diferentes tecnologías. Adicionalmente, entre algunos de los mecanismos implementados por este país para incentivar las ER, se encuentra la medición neta, la cual facilita la venta de excedentes por parte de los autogeneradores a pequeña, mediana y gran escala. Otros mecanismos son el impuesto al carbón y las subastas desde el año 2016; de estas últimas solo se han desarrollado 3, de las cuales 2 han sido en energía eólica y solar (Washburn \& Pablo-Romero, 2019).

\section{Colombia}

Su demanda de energías renovables en 2016 fue de 104,16 GWh. Al igual que en México, esta energía fue destinada, en su mayoría, para el sector residencial, que demandó el $45 \%$ de energía, seguido del sector industrial (33\%), el sector comercial (8\%), el sector transporte (7\%) y otros sectores, que demandaron el $7 \%$ (UPME, 2015a). Por su parte, el consumo de energía de fuentes renovables es liderado por la energía hidráulica, la cual presenta algunas externalidades por el uso del suelo y emisiones indirectas de $\mathrm{CO}_{2}$, seguido por la energía generada por biocombustibles sólidos como la madera, y con una pequeña participación, la generada por biogás, la solar fotovoltaica y la eólica (UPME, 2015a). 
Castaño-Gómez y García-Rendón: Análisis de los incentivos económicos en la capacidad...

El marco institucionalidad para el sector eléctrico en Colombia involucra varios organismos que se encargan de promover los incentivos. El encargado de diseñar las políticas en materia energética es el Ministerio de Minas y Energía. Por su parte, la Unidad de Planeación Minero Energética (UPME) es la encargada de la planeación y el apoyo para la formulación de políticas públicas. Asimismo, la encargada de regular el sector con el objetivo de asegurar la buena prestación del servicio es la Comisión de Regulación de Energía y Gas (CREG). De otro lado, la supervisión y el control del sector energético están a cargo de la Superintendencia de Servicios Públicos Domiciliarios y los aspectos judiciales a cargo de la Superintendencia de Industria y Comercio.

Fenómenos como el de principios de los noventa, cuando la escasez de agua generó un racionamiento de energía, reflejan la alta dependencia de los recursos hídricos, por lo que, cuando hay clima seco, se pone en riesgo el abastecimiento energético; en consecuencia, se han implementado diferentes mecanismos para incentivar el uso de FNCER. La Tabla 2 presenta un resumen de las normas establecidas para incentivar el crecimiento de la participación de este tipo de fuentes. Como puede observarse en esta tabla, es bastante la normatividad que existe relacionada con el tema, pero es la Ley 1715 la que establece las políticas para la adopción de FNCER y se convierte en el referente para el desarrollo de las tendencias que hoy observamos en materia de energía renovable, incluida la solar FV.

Tabla 2. Normativas para incentivar las FNCER en Colombia

\begin{tabular}{ll}
\hline Documento & Descripción \\
\hline Ley 142 de 1994 & $\begin{array}{l}\text { Define los esquemas generales de regulación que deben cumplir } \\
\text { las empresas de servicios públicos y el papel de las Comisiones } \\
\text { de Regulación, incluida la libre competencia y eficiencia en la } \\
\text { prestación de los servicios. }\end{array}$ \\
\hline Ley 143 de 1994 & $\begin{array}{l}\text { Establece lineamientos para la integración vertical y regula el } \\
\text { poder de mercado. Además, prohíbe explícitamente la entrega de } \\
\text { excedentes de generación a la red eléctrica para autogeneradores. }\end{array}$ \\
\hline
\end{tabular}


Tabla 2. Continuación

\begin{tabular}{|c|c|}
\hline Documento & Descripción \\
\hline $\begin{array}{l}\text { Resolución } 085 \text { de } 1996 \\
\text { (CREG) }\end{array}$ & $\begin{array}{l}\text { Reglamentan las actividades de cogenerador conectado al Siste- } \\
\text { ma Interconectado Nacional (SIN); posteriormente, la Resolu- } \\
\text { ción CREG-032/01 realiza modificaciones a esta resolución. }\end{array}$ \\
\hline $\begin{array}{l}\text { Resolución } 128 \text { de } 1996 \\
\text { (CREG) }\end{array}$ & $\begin{array}{l}\text { Limita la participación accionaria en el capital de una empresa } \\
\text { generadora o comercializadora, sobre una empresa distribuidora, } \\
\text { y viceversa. }\end{array}$ \\
\hline Ley 697 de 2001 & $\begin{array}{l}\text { Creó el Programa de Uso Racional y Eficiente de la Energía } \\
\text { (PROURE) y demás formas de Energías No Convencionales. }\end{array}$ \\
\hline $\begin{array}{l}\text { Resolución } 060 \text { de } 2007 \\
\text { (CREG) }\end{array}$ & $\begin{array}{l}\text { Establece normas sobre la participación en la actividad de } \\
\text { generación de energía eléctrica. }\end{array}$ \\
\hline Ley 1215 de 2008 & $\begin{array}{l}\text { Define la cogeneración como la producción combinada de } \\
\text { energía eléctrica y energía térmica que hace parte integrante de } \\
\text { su actividad productiva. }\end{array}$ \\
\hline $\begin{array}{l}\text { Resolución } 097 \text { de } 2008 \\
\text { (CREG) }\end{array}$ & $\begin{array}{l}\text { Aprueba los principios generales y la metodología para } \\
\text { el establecimiento de los cargos por uso del Sistemas de } \\
\text { Transmisión Regional (STR) y los Sistemas de Distribución } \\
\text { Local (SDL). }\end{array}$ \\
\hline Ley 1715 de 2014 & $\begin{array}{l}\text { Regula la integración de las energías renovables no convencio- } \\
\text { nales al sistema energético nacional y se establecen diferentes } \\
\text { incentivos. También permite la entrega de excedentes a la red. }\end{array}$ \\
\hline $\begin{array}{l}\text { Decreto } 2469 \text { de } 2014 \\
\text { (MME) }\end{array}$ & $\begin{array}{l}\text { Establecen los lineamientos de política energética en materia de } \\
\text { entrega de excedentes autogeneración. }\end{array}$ \\
\hline $\begin{array}{l}\text { Resolución } 038 \text { de } 2014 \\
\text { (CREG) }\end{array}$ & $\begin{array}{l}\text { Modifica el Código de Medida contenido en el anexo general del } \\
\text { Código de Redes. }\end{array}$ \\
\hline $\begin{array}{l}\text { Resolución } 281 \text { de } 2015 \\
\text { (UPME) }\end{array}$ & $\begin{array}{l}\text { Define el límite máximo de autogeneración a pequeńa escala a } 1 \\
\text { MW. }\end{array}$ \\
\hline $\begin{array}{l}\text { Resolución } 024 \text { de } 2015 \\
\text { (CREG) }\end{array}$ & Regula la actividad de autogeneración a gran escala. \\
\hline $\begin{array}{l}\text { Resolución } 227 \text { de } 2015 \\
\text { (CREG) }\end{array}$ & $\begin{array}{l}\text { Define la metodología para determinar la energía firme de } \\
\text { plantas solares FV. }\end{array}$ \\
\hline $\begin{array}{l}\text { Decreto } 348 \text { de } 2017 \\
\text { (MME) }\end{array}$ & $\begin{array}{l}\text { Estable los lineamientos de política pública en materia de gestión } \\
\text { eficiente de la energía y entrega de excedentes de autogeneración } \\
\text { a pequeña escala }(0,1 \mathrm{MW}) \text {. }\end{array}$ \\
\hline
\end{tabular}

Continúa 
Castaño-Gómez y García-Rendón: Análisis de los incentivos económicos en la capacidad...

Tabla 2. Continuación

\begin{tabular}{ll}
\hline Documento & Descripción \\
\hline $\begin{array}{l}\text { Resolución 030 de 2018 } \\
\text { (CREG) }\end{array}$ & $\begin{array}{l}\text { Regula las actividades de autogeneración a pequeña y gran } \\
\text { escala y de generación distribuida en el Sistema Interconectado } \\
\text { Nacional (SIN). }\end{array}$ \\
\hline $\begin{array}{l}\text { Resolución 038 de 2018 } \\
\text { (CREG) }\end{array}$ & $\begin{array}{l}\text { Establece las reglas para la actividad de autogeneración en las } \\
\text { Zonas No Interconectadas (ZNI). }\end{array}$ \\
\hline $\begin{array}{l}\text { Resolución 40072 de } \\
\text { 2018 (MME) }\end{array}$ & $\begin{array}{l}\text { Implementación de la Infraestructura en Medición Avanzada, } \\
\text { AMI por sus siglas en inglés, en el Sistema Interconectado } \\
\text { Nacional (SIN). }\end{array}$ \\
\hline $\begin{array}{l}\text { Resoluciones 40791 y } \\
\text { y0795 de 2018 (MME) }\end{array}$ & $\begin{array}{l}\text { Abre la convocatoria para la primera subasta de contratos de largo } \\
\text { plazo de energía eléctrica (a 10 ańos), la cual buscan incentivar } \\
\text { la instalación de FNCER. }\end{array}$ \\
\hline $\begin{array}{l}\text { Resoluciones 103 y 104 } \\
\text { de 2018 (CREG) }\end{array}$ & $\begin{array}{l}\text { Abre la subasta para la asignación de las Obligaciones de Energía } \\
\text { Firme del Cargo por Confiabilidad para el período comprendido } \\
\text { entre el 1 de diciembre de 2022 y el 30 de noviembre de 2023. }\end{array}$ \\
\hline $\begin{array}{l}\text { Resolución 098 de 2019 } \\
\text { (CREG) }\end{array}$ & $\begin{array}{l}\text { Establece las condiciones para la incorporación de sistemas de } \\
\text { almacenamiento con baterías en el SIN. }\end{array}$ \\
\hline $\begin{array}{l}\text { Resoluciones 40590 y } \\
40591 \text { de 2019 (MME) }\end{array}$ & $\begin{array}{l}\text { Establece los procedimientos para el desarrollo de subastas de } \\
\text { energía renovable no convencional, reemplazando la 40791 de } \\
\text { 2018. }\end{array}$ \\
\hline $\begin{array}{l}\text { Ley 1955 de 2019 } \\
\text { Establece que entre un 8 y 10\% de las compras de energía de los } \\
\text { comercializadores deben ser de fuentes de energía renovables no } \\
\text { convencionales. }\end{array}$ \\
\hline
\end{tabular}

Fuente: procesamiento propio.

Estas normas incluyen aspectos como la libre competencia para la actividad de generación, exenciones tributarias, la posibilidad de vender excedentes a la red a través de autogeneración, generación distribuida y subastas para incentivar los contratos de largo plazo con FNCER en Colombia. A pesar de que en la primera subasta que se realizó para FNCER en 2018, a partir de la Resolución 40791 de 2018 del MME, no se adjudicó ningún proyecto debido a que no se cumplieron los criterios de competencia, en la subasta del cargo por confiabilidad, realizada en marzo de 2019, parte de su adjudicación se hizo a FNCER: 6 proyectos eólicos y 2 solares, por 
1398 MW. Además, en la segunda subasta para contratos de largo plazo con FNCER, realizada en octubre de 2019 por medio de la Resolución 40590 de 2019 del MME, se adjudicaron 1298 MW de capacidad instalada, 5 proyectos de energía eólica y 3 solares. Esto representa aproximadamente el 11\% del total de la capacidad de generación del país.

En contraposición con los ideales de "dejar hacer" y "dejar pasar" de Smith (1794), en el sector energético colombiano existe amplia intervención estatal en cuanto al servicio de energía. Si bien se apoya en la Ley 142 de 1994, la cual regula los servicios públicos, la ley marco del sector eléctrico es la Ley 143 de 1994. Cabe aclarar que la Ley 142 se dio como resultado de un estado de emergencia económica y social sucedido entre finales de los años ochenta y principios de los noventa. En gran parte esta emergencia se atribuyó a la crisis energética que se dio en 1992, lo cual obligó a tomar medidas como el racionamiento del consumo de energía eléctrica (Moreno, 2017). La Ley 143 determina que los agentes creados posteriormente a su entrada en vigencia "no podrán realizar más de una de las actividades relacionadas con el mismo con excepción de la comercialización que puede realizarse en forma combinada con una de las actividades de generación o distribución" (Congreso de Colombia, 1994a).

Pero solo a partir de la Ley 1715 de 2014 se regula la integración de FNCER al sistema energético nacional, cuyo objetivo principal es la diversificación de la matriz energética del país, integrando tipos de generación de energía más limpias y sostenibles. Los incentivos en esta Ley para la implementación de estas fuentes son de tipo indirecto, como la reducción al impuesto de renta ( $50 \%$ del valor total realizado en la inversión), exención de IVA y aranceles (en el CAPEX de los proyectos) y depreciación acelerada de activos. Adicional a esto, se implementó la creación del Fondo de Energías No Convencionales y Gestión Eficiente de la Energía (FENOGE), con el que se busca financiar, impulsar y promocionar las soluciones de autogeneración y la mejora en la eficiencia energética. En la Figura 6, se presenta la estructura de esta Ley y los decretos y resoluciones expedidos por la CREG y que lo soportan. 
Castaño-Gómez y García-Rendón: Análisis de los incentivos económicos en la capacidad...

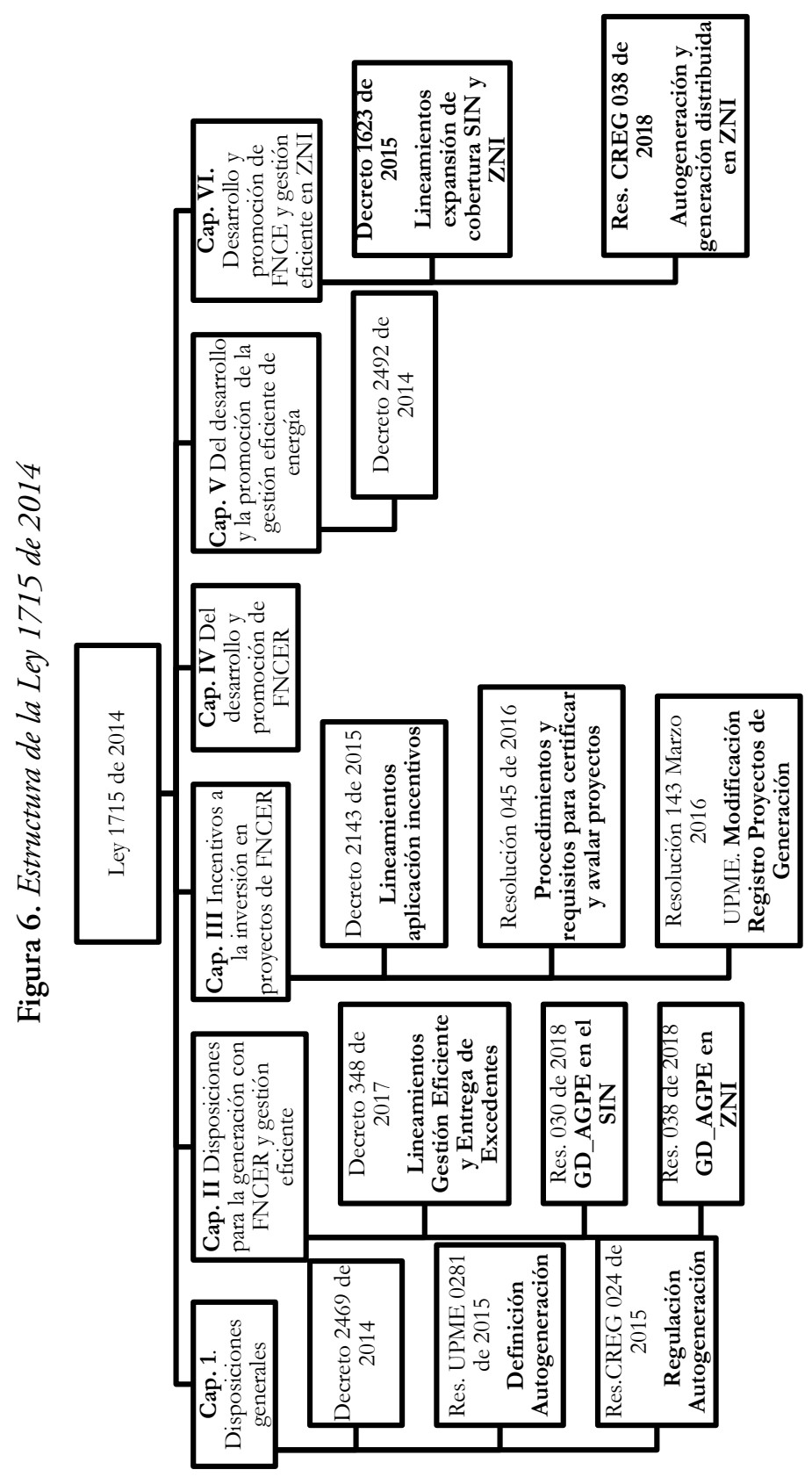

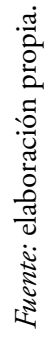


Estas políticas han estimulado el uso de fuentes alternativas para la generación de energía eléctrica. Gracias a esto, desde hace un par de años la tendencia viene cambiando; una prueba de esto se ve reflejada en el registro de proyectos vigentes de generación de la UPME. Como muestra la Figura 7, para 2018, 391 de los 575 proyectos inscritos son solares, con una participación del $68 \%$.

Figura 7. Participación de proyectos de generación en Colombia, 2018

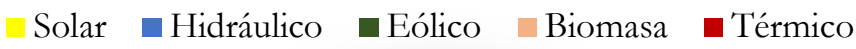

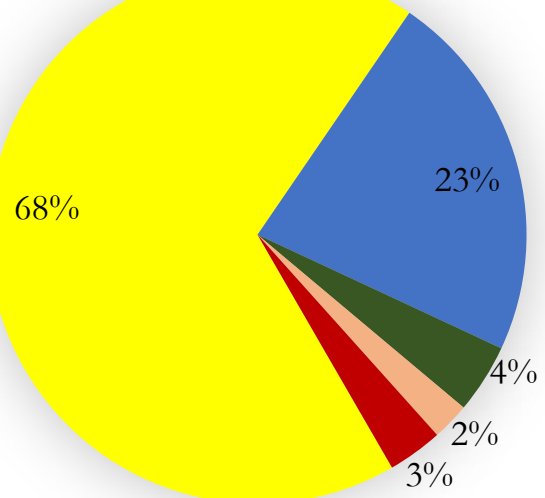

Fuente: elaboración propia a partir de UPME (2018a).

La Figura 8 presenta el contraste del total de proyectos solares FV inscritos y el registro de aplicación a incentivos de la Ley 1715. En 2018, 201 proyectos de un total de 232 participaron por incentivos de esta ley (UPME, 2018b). Aunque ambas tendencias son crecientes y tienen la misma pendiente, existe una brecha entre los proyectos registrados para la generación FV y los de la misma tecnología que aplican para incentivos de la Ley 1715. La causa de esta diferencia, en gran parte, son los trámites y costos transaccionales que implica la postulación a estos incentivos, ya que los proyectos deben ser registrados en la UPME y aprobados por la Autoridad Nacional de Licencias Ambientales (ANLA). Además, no todos los proyectos registrados son aprobados para acceder a los incentivos; por ejemplo, hasta el año 2018 habían aprobados 372, archivados 54, desistidos 22, en estudio 
Castaño-Gómez y García-Rendón: Análisis de los incentivos económicos en la capacidad...

46 y rechazados 24 (UPME, 2018b). Del total de proyectos de FNCER, los solares son los que mayor participación tienen en el registro y aplicación para estos incentivos, $\mathrm{y}$, desde que se tiene registro de los mismos, representan el $90 \%$ de ellos.

Figura 8. Evolución del registro de proyectos solares FVy el registro de estos a incentivos de la Ley 1715

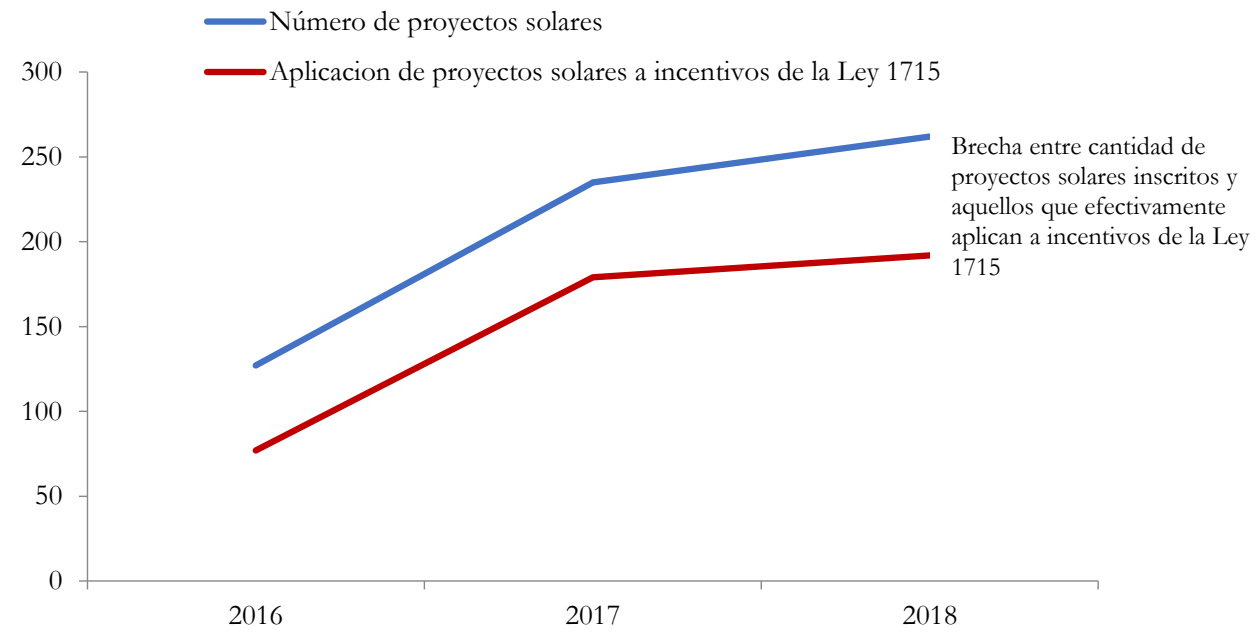

Fuente: elaboración propia a partir de UPME (2018a, 2018b).

\section{Metodología}

\section{A. Revisión de modelos}

En la revisión bibliográfica realizada, se encontró que diferentes modelos se han utilizado para evaluar la difusión de una tecnología o de un producto en un mercado específico. Entre los principales están: i) el de paridad tarifaria, ii) modelo basado en agentes y iii) modelo Bass. El primero, en el caso de sistemas fotovoltaicos distribuidos, se entiende como el momento en el que la tarifa de energía producida con esta fuente es más barata que la del operador de red o el comercializador (Konzen \& Zilles, 2014). Algunos de los problemas de este modelo consisten en que el hecho de que una tecnología 
sea más barata que otra no significa que vaya a ser adoptada en un mercado y, por tanto, estos modelos no proyectan realmente la capacidad de penetración de una tecnología en un mercado. Este es el caso concreto de los calentadores solares, los cuales ya han alcanzado la paridad tarifaria en varios países, pero su acogida en el mercado ha sido baja.

Una característica inherente del modelo basado en agentes es la calidad y cantidad de datos que hay que tener sobre la población de estudio y, dado que permite simular las interacciones estratégicas de los individuos en una población, es más utilizado en poblaciones menores, como lo utilizaron en sus estudios Palmer et al. (2015) y Zhao et al. (2011). Sin embargo, la cantidad de datos necesaria para modelarlo a una escala mayor hace muy compleja su implementación, especialmente con la tecnología solar FV, de la cual no se cuenta con buenas bases de datos para Colombia (Arias et al., 2019).

El tercer modelo es el desarrollado por Frank Bass en 1969 en su trabajo A new product growth for model consumer durables (Bass, 2004). Este autor considera la teoría de Difusión de la Innovación, desarrollado por Rogers (1962), quien clasifica a los individuos en las siguientes categorías: innovador, adoptante, temprano, mayoría temprana, mayoría tardía y rezagado. Esta es una alternativa interesante que hace una segmentación de los agentes de acuerdo al momento en el que adoptan un nuevo producto y permite realizar pronósticos de demanda de nuevas tecnologías (Jiang et al., 2006).

La principal ventaja de este modelo de difusión es su simplicidad, ya que requiere menor cantidad de datos de entrada. Así, el modelo que utilizamos en este estudio para estimar el crecimiento de la energía solar FV en Colombia es el utilizado por Bass. A pesar de que una de las limitaciones es la definición de los parámetros para los coeficientes de innovación y de imitación, ya han sido realizados diferentes estudios que estiman los valores que pueden tomar estas variables, los cuales utilizamos en nuestro estudio.

\section{B. Modelo de Bass}

Bass considera coeficientes de imitación, que se refieren a la probabilidad de que un imitador adopte un nuevo producto, y el coeficiente de innovación, 
Castaño-Gómez y García-Rendón: Análisis de los incentivos económicos en la capacidad...

asociado a la probabilidad de que un innovador adquiera un producto o servicio. Ambos coeficientes son la base para identificar el número de adoptantes de una tecnología. Este modelo es uno de los más referenciados en la literatura de marketing para pronosticar la difusión y demanda de tecnologías. El modelo tiene la forma funcional representada por la ecuación (1).

$$
\frac{f(t)}{1-F(t)}=p+\frac{q}{m} N(t),
$$

donde, $f(t)$ es la probabilidad de adopción en un tiempo $t ; F(t)$ es la distribución acumulada; $p$ es el coeficiente de innovación; $q$ es el coeficiente de imitación; $m$ es el mercado potencial final, en nuestro caso, la capacidad total instalable de energía solar; y $N(t)$ es el número acumulado de adoptantes, para nuestro caso, la potencia acumulada de generación solar.

Además, la ecuación (2) complementa la estimación de una función de demanda de la ecuación (1) alterada por un factor, pero teniendo en cuenta el potencial del mercado como lo establecen Cardoso et al. (2007):

$$
N(t)=m F(t) .
$$

En la Figura 9, puede observarse el esquema de la metodología donde se relacionan la rentabilidad de la tecnología que varía teniendo en cuenta los incentivos, el indicador que se utiliza para medir la rentabilidad es el tiempo de retorno de la inversión y este, a su vez, determina la fracción máxima de mercado, para ingresar al modelo de Bass y modelar escenarios de crecimiento de la tecnología solar FV en Colombia.

\section{Mercado potencial (m)}

Para aproximarnos a un mercado potencial, primero es necesario analizar las diferentes variables que influyen al momento de que un adoptante de una tecnología decida invertir en esta. Se toma como premisa que la forma en que evalúan la decisión de invertir es mediante un análisis financiero previo. Hay muchas métricas de rendimiento financiero disponibles, incluido el tiempo de retorno de la inversión (simple o descontado), el valor presente neto, la 


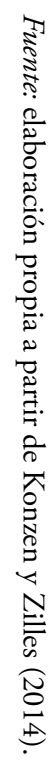

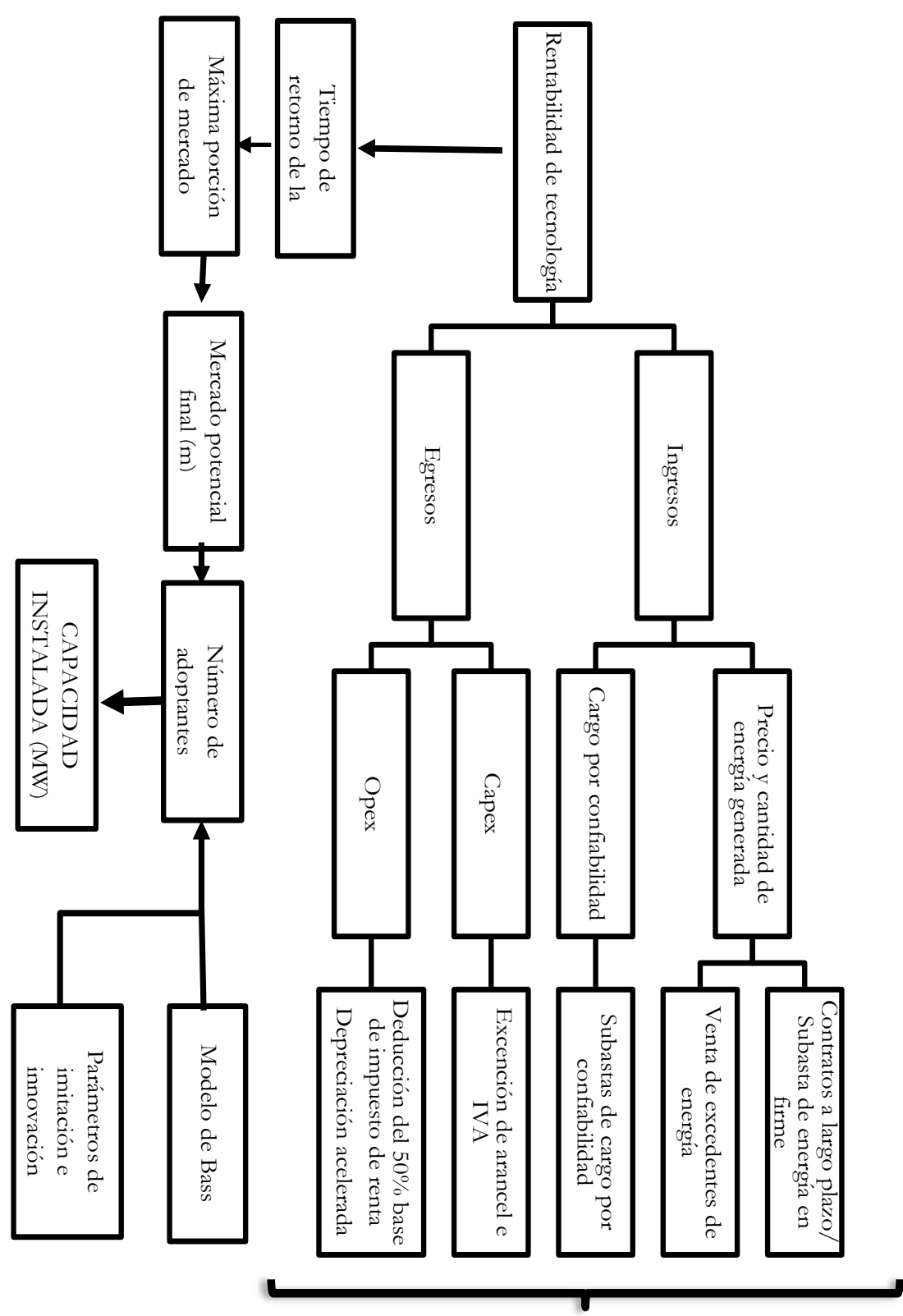

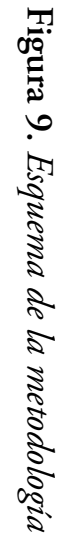

SOJIWONOJH SOAILNGJNI 
Castaño-Gómez y García-Rendón: Análisis de los incentivos económicos en la capacidad...

relación costo-beneficio y el costo nivelado de la electricidad. La métrica utilizada en un análisis dado depende, en gran medida, del tipo de consumidor: mientras que los clientes residenciales pueden usar métricas relativamente simples - como el tiempo de retorno de la inversión- para decidir invertir en un sistema FV, los clientes comerciales, por su parte, pueden usar métricas más sofisticadas para dar cuenta de la depreciación del capital (Denholm et al., 2009).

La fracción de usuarios dispuestos a adoptar la tecnología de acuerdo con el valor del tiempo de retorno de la inversión simple fue presentada por Beck (2009), y se representa por la ecuación (3):

$$
f w a=e^{-S T P * P T}
$$

donde, $S T P$ es la sensibilidad al tiempo de retorno de la inversión y PT es el tiempo de retorno de la inversión.

El valor de la sensibilidad del tiempo de retorno de la inversión determina la forma de la curva de fracción de mercado máxima y el usuario puede configurarla para caracterizar diferentes dinámicas del mercado FV y realizar análisis de sensibilidad de la conducta. Beck (2009) y Denholm et al. (2009), Navigant Consulting (2007), y Kastovick et al. (1982) utilizan un valor de 0,3 ; en este estudio tomamos el mismo valor. Además, utilizamos la función de probabilidad acumulada de la ecuación (4), propuesta por Mahajan et al. (1990):

$$
F(t)=\frac{1-e^{-(p+q) t}}{1+\frac{q}{p} e^{-(p+q) t}},
$$

donde, $p$ corresponde al coeficiente de innovación y $q$ al de imitación; como sabíamos anteriormente, la adopción acumulada en el tiempo $t$ es $m F(t)$.

Para hallar la función de probabilidad acumulada se deben definir los coeficientes de innovación e imitación, los cuales varían dependiendo de si se aplican incentivos, ya que estos aumentan la probabilidad de adopción de una tecnología. Para cada escenario se toman los coeficientes como se muestra en la Tabla 3.

Para el mercado potencial analizamos diferentes estudios, por ejemplo, el Ministerio de Minas y Energía calcula que solo en el departamento de la 
Guajira se tiene un potencial solar cercano a los 42 GW (MME, 2018b), pero solo se limita a esta región, y no discrimina las áreas que no son aprovechables; tampoco suministra información de cuánto se genera por grandes proyectos en generación distribuida, por tanto, no es una buena aproximación del potencial total del país. Otro estudio corresponde al realizado por la UPME, que calcula un potencial solar FV estimado en techos para las principales ciudades del país, dando como resultado un potencial cercano a 1,88 GW (UPME, 2018a), el cual es el mercado potencial para nuestro análisis.

Tabla 3. Supuestos coeficientes de innovación (p) e imitación (q) para cada escenario

\begin{tabular}{lcc}
\hline Escenario & Coeficiente $\mathbf{p}$ & Coeficiente q \\
\hline Incentivos de la Ley 1715 Residencial & 0,002 & 0,4 \\
Incentivos de la Ley 1715 Comercial & 0,002 & 0,4 \\
Incentivos de la Ley 1715 Granja Solar & 0,002 & 0,4 \\
\hline
\end{tabular}

Fuente: elaboración propia a partir de Denholm et al. (2009).

Cabe anotar que la metodología utilizada por la UPME consiste en un modelo econométrico de largo plazo, que combina pronósticos a través de modelos multivariados como los VAR (modelo de vectores autorregresivos) y los VEC (modelo de vectores de corrección de error), los cuales proponen un sistema de ecuaciones, con tantas ecuaciones como series a analizar o a predecir UPME (2017).

\section{Resultados}

Se estiman tres escenarios para hacer una proyección de la capacidad instalada de energías solar FV en Colombia a 2030, teniendo en cuenta los incentivos vigentes y la implementación de los mismos. El planteamiento de estos tres escenarios obedece, de un lado, a la disponibilidad de información que se pudo conseguir a partir del MME y de la UPME y, por otra parte, a que, acorde a las características y el funcionamiento del sector eléctrico, considere tres casos típicos que pudieran plantearse en Colombia. El primero se refiere a un proyecto residencial, el segundo a la implementación de una granja solar y, el último, a un proyecto a gran escala, el cual puede considerarse como el 
Castaño-Gómez y García-Rendón: Análisis de los incentivos económicos en la capacidad...

escenario más realista dado el potencial que se tiene en el país a partir de la energía solar. Como se ha descrito en el texto, la acogida de esta tecnología no depende únicamente de sus costos de generación, sino de los incentivos generados por medio de la regulación que permitan autogenerar e inyectar los excedentes a la red (generación distribuida) o simplemente por medio de la autogeneración.

\section{A. Escenarios}

Se toma como referencia el estudio de Integración de Energías Renovables propuesto por la UPME (2017), en el cual se realiza una evaluación costo-beneficio de los proyectos de FNCER con el impacto de los incentivos de la Ley 1715. Como se dijo en el párrafo anterior, se presentan 3 escenarios, cuyas características específicas se muestran en la Tabla 4. También se considera un escenario base sin aplicar a beneficios tributarios de la ley, en el que se evalúan solo los ahorros tributarios por no conectarse a la red, pero no se logra la difusión de la tecnología y, por tanto, no se incluye en este escrito. El escenario 1 presenta un proyecto residencial de capacidad típica al cual, otorgándole beneficios de la Ley 1715, se le conceden los incentivos de exención de IVA y arancel al costo nivelado de la energía. Este escenario es rentable con entrega de excedentes y esquemas de créditos para los excedentes que sean producidos por autogeneradores de pequeña escala que utilicen FNCER (UPME, 2017). Las principales características de este escenario es que se vende el $49 \%$ de la energía generada en el sistema, se considera la existencia de un medidor bidireccional, además se incluye un esquema de medición neta y el precio de bolsa para la remuneración de la energía transada en el sistema.

En el escenario 2, el cual consiste en una granja solar, al costo nivelado de la energía se le aplica los incentivos de exención de IVA y arancel y la venta de excedentes y deducción de renta, aplicando a nivel residencial la tarifa con contribución para superar el LCOE. Se plantea un modelo financiero de una granja solar FV con una capacidad instalada de $375 \mathrm{~kW}$. Está basado en el cálculo de los gastos de capital (CAPEX), los cuales hacen referencia a la financiación utilizada por la empresa para conseguir activos físicos o 
actualizar activos, teniendo en cuenta los gastos de mantenimiento y los gastos de expansión. Asimismo, se identifican los diferentes gastos de operación (OPEX) y, adicionalmente, se determinó el promedio ponderado del costo de capital (WACC, por sus siglas en inglés) (9,7\%) del proyecto teniendo una estructura de capital del $80 \%$ de patrimonio y un $20 \%$ de deuda. El precio utilizado es $175 \mathrm{USD} / \mathrm{MWh}$ proyectado con el índice de precios al productor (IPP) y el LCOE es de 190 USD/MWh.

Tabla 4. Supuestos financieros para la elaboración de escenarios

\begin{tabular}{|c|c|c|c|c|c|c|}
\hline Escenario & $\begin{array}{l}\text { Potencia } \\
\text { Instalada }\end{array}$ & $\begin{array}{c}\text { LCOE } \\
\text { (USD/MWh) }\end{array}$ & $\begin{array}{c}\text { Precio de la } \\
\text { energía } \\
\text { (USD/MWh) }\end{array}$ & WACC & TIR (\%) & $\begin{array}{l}\text { Tiempo de } \\
\text { recuperación } \\
\text { de la } \\
\text { inversión } \\
\text { (Años) }\end{array}$ \\
\hline $\begin{array}{l}\text { 1. Incentivos de la Ley } \\
\text { 1715, Residencial }\end{array}$ & $3 \mathrm{kWp}$ & 193 & 211 & $4 \%$ (Sin deuda) & 11,20 & 8,8 \\
\hline $\begin{array}{l}\text { 2. Incentivos de la Ley } \\
\text { 1715, Granja Solar }\end{array}$ & $375 \mathrm{kWp}$ & 190 & 175 & $\begin{array}{l}9,02 \%(20 \% \\
\text { deuda) }\end{array}$ & 9,7 & 10 \\
\hline $\begin{array}{l}\text { 3. Gran escala Incenti- } \\
\text { vos Ley } 1715\end{array}$ & 5,8 MW & 189 & 175 & $\begin{array}{l}9,02 \%(20 \% \\
\text { deuda })\end{array}$ & 30,47 & 4 \\
\hline
\end{tabular}

Nota: $p$ se refiere a horas pico. En ninguno de los escenarios se aplica el cargo por confiabilidad, pues se tiene en cuenta que ninguno de los proyectos es mayor a $20 \mathrm{MW}$.

Fuente: elaboración propia.

El escenario 3 considera un proyecto a gran escala, es decir, con una capacidad instalada mayor a $1 \mathrm{MW}$. Para el caso de estudio es de 5,8 MW; también se evalúa con la metodología de flujo de caja descontado, utilizando un LCOE de 189 USD/MWh e incluye los incentivos. La tasa interna de retorno (TIR) del proyecto es 30,47\% y tiene un precio de energía de $175 \mathrm{USD} / \mathrm{MWh}$ indexado con IPP para realizar la proyección. En este caso, el periodo de retorno de la inversión es de 4 años, el más corto de todos los escenarios planteados.

\section{B. Resultados de los escenarios}

Los resultados obtenidos para los tres escenarios se presentan en la Tabla 5. Para el escenario 1 con autogeneración y venta de excedentes a la red, coincide con los resultados pronosticados por la UPME en su Plan de 
Castaño-Gómez y García-Rendón: Análisis de los incentivos económicos en la capacidad...

Expansión (Resolución 40072 de 2018 del MME). Debe tenerse en cuenta que, para su cierre financiero, este escenario debe aplicar a los beneficios tributarios y exenciones de la Ley 1715 de 2014. En el escenario 2, suponiendo como potencial utilizable para granjas solares FV $3000 \mathrm{MW}$, se determina que al 2030 la capacidad instalada para granjas solares distribuidas es de 182 MW.

En la Figura 10 se observa la difusión de esta tecnología para el caso de energía distribuida, tanto a nivel residencial (representado por la línea azul) como para granjas solares (representado por la línea roja). A pesar de que las metodologías utilizadas para el pronóstico son diferentes alcanza valores cercanos a los pronosticados en el Plan de Expansión (Resolución 40072 de 2018 del MME).

Tabla 5. Resultados de los escenarios

\begin{tabular}{ccccccc}
\hline \multirow{2}{*}{ Año } & \multicolumn{3}{c}{ Capacidad Instalada $[\mathrm{MW}]$} & \multicolumn{3}{c}{ Tasa de crecimiento (\%) } \\
\cline { 2 - 7 } & Escenario 1 & Escenario 2 & Escenario 3 & Escenario 1 & Escenario 2 & Escenario 3 \\
\hline 2019 & 29 & 21 & 31 & - & 44 & 69 \\
2020 & 39 & 30 & 52 & 36 & 40 & 60 \\
2021 & 51 & 43 & 83 & 31 & 36 & 54 \\
2022 & 64 & 58 & 128 & 26 & 31 & 50 \\
2023 & 78 & 76 & 193 & 21 & 26 & 47 \\
2024 & 91 & 95 & 283 & 16 & 21 & 44 \\
2025 & 102 & 115 & 407 & 12 & 16 & 40 \\
2026 & 111 & 134 & 569 & 9 & 12 & 36 \\
2027 & 117 & 150 & 773 & 6 & 9 & 31 \\
2028 & 123 & 164 & 1012 & 4 & 6 & 26 \\
2029 & 126 & 174 & 1275 & 3 & 4 & 21 \\
2030 & 129 & 182 & 1542 & 2 & 3 & 16 \\
\hline
\end{tabular}

Fuente: elaboración propia.

Los resultados del escenario 3, un proyecto a gran escala es el más rentable y el de mayor potencial de crecimiento, pues llega a una capacidad de 1542 MW en 2030, un poco más si se compara con los pronósticos del Plan de Expansión (Resolución 40072 de 2018 del MME) en el que, siendo un poco más conservador, se plantean $1090 \mathrm{MW}$ instalados para ese mismo año.

La Figura 11 presenta la difusión de tecnologías para el escenario más rentable, el cual, al igual que los anteriores, presenta un comportamiento en S, 
pero en este caso con un crecimiento mayor dado que se trata de un escenario a gran escala, teniendo en cuenta el potencial solar utilizable en Colombia.

Figura 10. Resultados de difusión de los escenarios 1 y 2

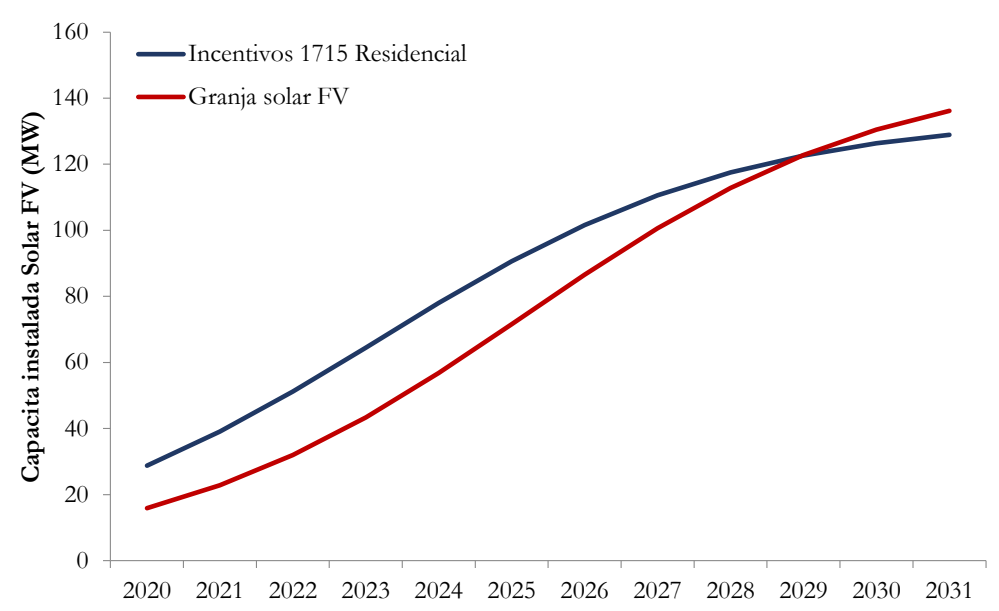

Fuente: elaboración propia.

Figura 11. Resultados de difusión del escenario 3

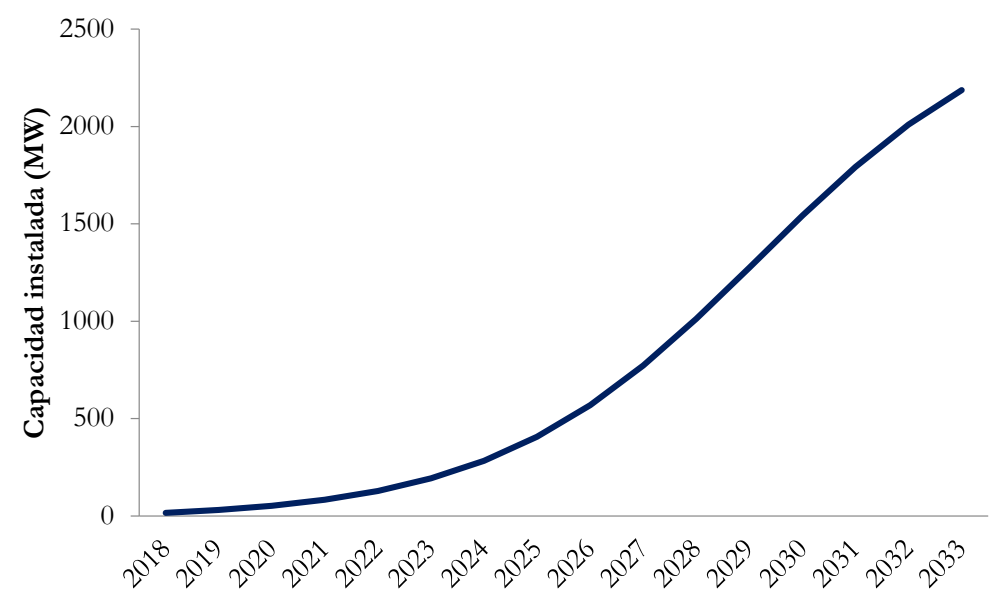

Fuente: elaboración propia. 
Castaño-Gómez y García-Rendón: Análisis de los incentivos económicos en la capacidad...

\section{Conclusiones}

A partir de la revisión de literatura (sección I), específicamente en las secciones A y C, donde se puede evidenciar la caída sustancial que han presentado los costos nivelados de las FNCER, la solar y la eólica en los mercados eléctricos internacionales, lo que, a su vez, ha permitido la utilización de mecanismos de mercado más eficientes, como las subastas, para la financiación de inversión en estas fuentes de generación. Así lo demuestra la segunda subasta realizada por el MME, en octubre de 2019, para la realización de contratos de largo plazo que incentiven la incorporación de FNCER. Por medio de la Resolución 40590 de 2019 del MME se adjudicaron 1298 MW de capacidad instalada en 5 proyectos de energía eólica y 3 solares, lo cual representa, aproximadamente, el $5 \%$ del total de la capacidad de generación del país y, que sumado a los 1398 MW de 6 proyectos eólicos y 2 solares -adjudicados en la subasta de energía firme del cargo por confiabilidad, realizada en marzo de 2019- representa cerca del $11 \%$ de total de la capacidad de generación en Colombia con FNCER. Estos proyectos se empezarán a implementar a partir de 2022.

Además, a nivel regulatorio se han dado cambios importantes en Colombia para la integración de FNCER, como se puede evidenciar a partir de la Tabla 2. El punto de referencia lo constituye la Ley 1715 de 2014, la cual determina los incentivos fiscales, como reducción al impuesto de renta, exención de IVA y aranceles y depreciación acelerada de activos. Las resoluciones 024 de 2015 y 030 de 2018 del CREG, definen la regulación de las actividades de generación distribuida y autogeneración a pequeña y gran escala en el SIN y, la Resolución 038 de 2018 del CREG, define las reglas para la actividad de autogeneración en ZNI. Esto representa una alternativa viable para la diversificación de la matriz energética del país y, por tanto, garantizar la confiablidad en el suministro. No obstante, quedan retos por resolver que incentiven la adopción de estas tecnologías. Por ejemplo, se deben establecer mecanismos para disminuir los costos transaccionales, ya que en la actualidad existe gran reproceso, dado el alto número de trámites que se requieren para acceder a los beneficios tributarios de las leyes 1715 de 2014 y 1955 de 2019. 
Con respecto a los resultados de los escenarios obtenidos a partir del modelo de Bass, en general, estos son conservadores comparativamente con países de la región. El más realista es el escenario para proyectos a gran escala, el cual incorpora los incentivos establecidos por la Ley 1715; también es el más rentable y el de mayor potencial de crecimiento, pues muestra una capacidad instalada de 1542 MW en 2030. Es importante aclarar que este no incluye el mecanismo de subasta y, como se dijo en la primera conclusión, esta es una buena alternativa que ayudará a la expansión de la capacidad instalada por medio de las FNCER, como lo evidenciaron los resultados a partir de la segunda subasta realizada en octubre de 2019 para incentivar los contratos de largo plazo.

Además, si bien no es el objeto de este estudio, con la promulgación de la Resolución 098 de 2019 de la CREG se da un paso importante respecto al almacenamiento, ya que define las condiciones para la incorporación de Sistemas de Almacenamiento con Baterías en el SIN. Con esta resolución se deja abierta la posibilidad de utilizar sistemas de almacenamiento para solucionar los problemas de las restricciones de los servicios complementarios para abastecer la demanda en términos reales. Estos sistemas pueden ser menos costosos para prestar estos servicios y, en el caso colombiano, pueden ser viables en regiones como la costa, ya que, la falta de inversiones en las redes de transmisión y distribución, hace que algunas plantas térmicas sean necesarias (monopolios locales) para garantizar la confiabilidad en la prestación de servicio.

\section{Agradecimientos}

Los autores agradecen a la Universidad EAFIT por apoyar esta investigación a través de la financiación del proyecto 828-000134. Esta investigación también se desarrolló en el marco del Programa de Investigación "ENERGETICA 2030", con el código 58864 de la iniciativa "Colombia Científica", el cual fue financiado por el Banco Mundial a través de la convocatoria "778-2017 Ecosistemas Científicos" y administrado por el Ministerio de Ciencia, Tecnología e Innovación - MinCiencias. 
Castaño-Gómez y García-Rendón: Análisis de los incentivos económicos en la capacidad...

\section{Referencias}

Arias, J., Carvajal, S. X. \& Arango, S. (2019). Understanding dynamics and policy for renewable energy diffusion in Colombia. Renewable Energy, 139, 1111-1119. https://doi.org/.1037//0033-2909.I26.1.78

Bass, F. M. (2004). A new product growth model for consumer durables. Management Science, 50(12), 1825-1832. https://www.jstor.org/stab le/30046153

Beck, R. W. Inc. (2009). Distributed renewable energy operating impacts and valuation study. https://appsrv.pace.edu/VOSCOE/?do=viewFullReso urce\&resID=J8PAM033116121012

Black \& Veatch (2015). Energy market perspectives. https://es.slideshare.net/ blackveatch/black-veatch-energy-market-perspective-2015-outlook

Böhringer, Ch., Cuntz, A., Harhof, D. \& Asane-Otoo, E. (2017). The impact of the German feed-in tariff scheme on innovation: Evidence based on patent filings in renewable energy technologies. Energy Economics, 67, 545-553. https://doi.org/10.1016/j.eneco.2017.09.001

Boyce, J. (2018). Carbon pricing: Effectiveness equity. Ecological Economics, 150, 52-61, 2018.

Bugrahan, A., Ugur, K., \& Bulent, S. (2011). The role of legislations and incentives in the growth of PV market in a developing country. 2017 International Renewable and Sustainable Energy Conference (IRSEC), 1-6. doi: 10.1109/IRSEC.2017.8477329

Bullard, N. (2018). Approaches for using scenarios in strategic decision making the future of energy. https://www.fsb-tcfd.org/wp-content/uploads/2018 /03/Presentation-Bloomberg-New-Energy-Finance.pdf

Cardoso, G. Andrade, I., Midori, C \& Moura, G. (2007). Modeling the acceptance and use of telecenters in Brazil. Journal of Technology Management \& Innovation, 2(4), 86-97. 
Congreso de Colombia (1994a, 11 de julio). Ley 143 de 1994 - Ley Eléctrica. Diario Oficial No. 41.434. http://www.secretariasenado.gov.co/senado /basedoc/ley_0143_1994.html

Congreso de Colombia (1994b, 11 de julio). Ley 142 de 1994 - Ley de servicios publicos domiciliarios. Diario Oficial No. 41.433. http://www.secretaria senado.gov.co/senado/basedoc/ley_0142_1994.html

Congreso de Colombia (2001, 3 de octubre). Ley 697 de 2001. Mediante la cual se fomenta el uso racional y eficiente de la energía, se promueve la utilización de energías alternativas y se dictan otras disposiciones. Diario Oficial No. 44.573. http://www.secretariasenado.gov.co/senado/based oc/ley_0697_2001.html

Congreso de Colombia (2008, 16 de julio). Ley 1215 de 2008. Por la cual se adoptan medidas en materia de generación de energía eléctrica. Diario Oficial No. 47.052. http://www.secretariasenado.gov.co/senado/based oc/ley_1215_2008.html

Congreso de Colombia (2014, 13 de mayo). Ley 1715 de 2014. Por medio de la cual se regula la integración de las energias renovables no convencionales al sistema energético nacional. http://wsp.presidencia.gov.co/Normativa /Leyes/Documents/LEY1715DEL13DEMAYODE2014.pdf

Congreso de Colombia (2019, 25 de mayo). Ley 1955 de 2019. Por la cual se expide el Plan Nacional de Desarrollo 2018 - 2022. Diario Oficial No. 50.964. http://www.secretariasenado.gov.co/senado/basedoc/ley_195 5_2019.html

CREG (Comisión de Regulación de Energía y Gas) (1996a, 15 de octubre). Resolución 085 de 1996. Por la cual se reglamentan las actividades del Cogenerador conectado al Sistema Interconectado Nacional (SIN). http: //apolo.creg.gov.co/Publicac.nsf/Indice01/Resoluci\%C3\%B3n-19 96-CRG85-96

CREG (1996b, 17 de diciembre). Resolución 128 de 1996. Por la cual se dictan reglas sobre la participación en las actividades de generación, distribución y comercialización de electricidad y se fijan límites a la participación 
Castaño-Gómez y García-Rendón: Análisis de los incentivos económicos en la capacidad...

accionaria entre empresas con actividades complementarias. http://apolo. creg.gov.co/PUBLICAC.NSF/Indice01/Resoluci\%C3\%B3n-1996-C R128-96

CREG (2007, 28 de junio). Resolución 060 de 2006. Por la cual se dictan normas sobre la participación en la actividad de generación de energía eléctrica. http://apolo.creg.gov.co/Publicac.nsf/Indice01/Resoluci $\%$ C3\%B3n-2007-CREG060-2007

CREG (2008, 26 de septiembre). Resolución 097 de 2008. Por la cual se aprueban los principios generales y la metodología para el establecimiento de los cargos por uso de los Sistemas de Transmisión Regional y Distribución Local. http://apolo.creg.gov.co/Publicac.nsf/Indice01/Resolucion-200 8-Creg097-2008

CREG (2014, 20 de marzo). Resolución 038 de 2014. Por la cual se modifica el Código de Medida contenido en el Anexo general del Código de Redes. http://apolo.creg.gov.co/Publicac.nsf/1c09d18d2d5ffb5b05256eee007 09c02/0131f0642192a5a205257cd800728c5e?OpenDocument

CREG (2015a, 13 de marzo). Resolución 024 de 2015. Por la cual se regula la actividad de autogeneración a gran escala en el sistema interconectado nacional (SIN) y se dictan otras disposiciones. http://apolo.creg.gov.co/Pu blicac.nsf/1c09d18d2d5ffb5b05256eee00709c02/67513914c35d6b8c 05257e2d007cf0b0/pprotect \UUtextdollarFILE/Creg024-2015.pdf

CREG (2015b, 11 de diciembre). Resolcuión 227 de 2015. Por la cual se define la metodología para determinar la energia firme de plantas solares fotovoltaicas. http://apolo.creg.gov.co/Publicac.nsf/1c09d18d2d5ffb5 b05256eee00709c02/59aa7fe361aca6c405257f39007956fe?OpenDoc ument

CREG (2018a, 30 de julio). Resolución 104 de 2018. Por la cual se fija la oportunidad para llevar a cabo la Subasta para la asignación de las Obligaciones de Energía Firme del Cargo por Confiabilidad para el periodo comprendido entre el $1^{\circ}$ de diciembre de 2022 y el 30 de noviembre de 2023. http://legal.legis.com.co/document/Index?obra=legcol\&document=le gcol_72c9efb38b0d472c8ebaf569a035caa6 
CREG (2018b, 26 de febrero). Resolución 030 de 2018. Por la cual se regulan las actividades de autogeneración a pequeña escala y de generación distribuida en el Sistema Interconectado Nacional. http://apolo.creg.gov.co/Publicac .nsf/1c09d18d2d5ffb5b05256eee00709c02/83b41035c2c4474f0525 8243005a1191? OpenDocument

CREG (2018c, 4 de mayo). Resolución 038 de 2018. Por la cual se regula la actividad de autogeneración en las zonas no interconectadas y se dictan algunas disposiciones sobre la generación distribuida en las zonas no interconectadas. https://app.vlex.com/\#vid/716634677

CREG (2019a, 20 de junio). Resolución 060 de 2019. Por la cual se hacen modificaciones y adiciones transitorial al Reglamento de Operación para permitir la conexión y operación de plantas solares fotovoltaicas y eólicas en el SIN y se dictan otras disposiciones. http://apolo.creg.gov.co/Publicac.n sf/1c09d18d2d5ffb5b05256eee00709c02/ca640edbe4b7b510052584 $2 \mathrm{~d} 0053745 \mathrm{~d} /$ protectlTU ItextdollarFILE/Creg060-2019.pdf

CREG (2019b, 6 de septiembre). Resolución 098 de 2019. Por la cualse definen los mecanismos para incorporar sistemas de almacenamiento con el propósito de mitigar inconvenientes presentados por la falta o insuficiencia de redes de transporte de energía en el SIN. https://2019.vlex.com/\#vid/811528253

Denholm, P., Drury, E. \& Margolis, R. (2009). The solar deployment system (SolarDS) model?: documentation and sample results (Technical Report, NREL/TP-6A2-45832). https://www.nrel.gov/docs/fy10osti/45832 .pdf

Dos Santos Carstens, D. D. \& da Cunha, S. K. (2019). Challenges and opportunities for the growth of solar photovoltaic energy in Brazil. Energy Policy, 125, 396-404. https://doi.org/10.1016/j.enpol.2018 .10 .063

Femin, V., Najmu, H., Dayana, K. B., Petra, I. \& Mathew, S. (2016). Financial incentive mechanisms for residential PV systems: an analysis based on the real performance data. 2016 International Conference on Cogeneration, Small Power Plants and District Energy (ICUE), 1-5. https: //doi.org/10.1109/COGEN.2016.7728963 
Castaño-Gómez y García-Rendón: Análisis de los incentivos económicos en la capacidad...

Hille, E., Althammer, W. \& Diederich, H. (2020). Environmental regulation and innovation in renewable energy technologies: Does the policy instrument matter? Technological, Forecasting \& Social Change, 153, 119921. https://doi.org/10.1016/j.techfore.2020.119921

Hunt, T. (2014). Swanson's Law and Making US Solar Scale Like Germany. https://www.greentechmedia.com/articles/read/is-there-really-a-swans ons-law

IRENA (2019). Renewable capacity statistics 2019. https://www.irena.org/pu blications/2019/Mar/Renewable-Capacity-Statistics-2019

Jiang, Z., Bass, F. M. \& Isaacson, P. (2006). Virtual Bass Model and the left-hand data-truncation bias in diffusion of innovation studies. International Journal of Research in Marketing, 23(1), 93-106. https: //doi.org/10.1016/j.ijresmar.2006.01.008

Kastovich, J.C., Lawrence, R.R., Hoffman, R.R. \& Pavlak, C. (1982). Advanced electric-heat-pump market and business analysis. Final Report. https://www.researchgate.net/publication/255526994_Advanced_elect ric-heat-pump_market_and_business_analysis_Final_report

Konzen, G. \& Zilles, R. (2014). Difusão de sistemas fotovoltaicos residenciais conectados ã rede no Brasil: uma simulaçáo via modelo de Bass. Programa de pós-graduação em energia. http://lsf.iee.usp.br/sites/default/files/Disse rtacao_Gabriel_Konzen.pdf

Laffont, J. \& Tirole, J. (1993). A theory of incentives in procurement and regulation. London: The MIT Press.

Mahajan, V., Muller, E. \& Bass, F.M. (1990). New product diffusion models in marketing: A review and directions for research. Journal of Marketing, 54(1), 1-26. https://doi.org/10.2307/1252170

Mints, P. (2011). Changing incentive structures and photovoltaic demand. 2011 37th IEEE Photovoltaic Specialists Conference, 3264-3268. https: //doi.org/10.1109/PVSC.2011.6186634 
MME (Ministerio de Minas y Energía) (2014, 2 de diciembre). Decreto 2469 de 2014. Por el cual se establecen los lineamientos de politica energética en materia de entrega de excedentes de autogeneración. https://www.minene rgia.gov.co/documents/10180/23517/36864-Decreto-2469-02Dic20 14.pdf

MME (2017, 1 de marzo). Decreto 348 de 2017. Por el cual se adiciona el Decreto número 1073 de 2015, en lo que respecta al establecimiento de los lineamientos de politica pública en materia de gestión eficiente de la energía y entrega de excedentes de autogeneración a pequeña escala. https: //dapre.presidencia.gov.co/normativa/normativa/DECRETO\%20348 \%20DEL\%2001\%20DE\%20MARZO\%20DE\%202017.pdf

MME (2018a, 29 de enero). Resolución 40072 de 2018. Por la cual se establecen los mecanismos para implementar la Infraestructura de Medición Avanzada en el servicio público de energía eléctrica. http://legal.legis.com.co/docum ent/Index?obra=legcol\&document=legcol_d9dbab376fb849659b43f8a $1 \mathrm{ca} 96 \mathrm{~b} 435$

MME (2018b). Política pública para el sector eléctrico. https://www.energiam ayorista.com.co/wp-content/uploads/2018/11/2018-11-02-10-15-Poli tica-Publica-Ministerio-de-Minas-y-Energia.pdf

MME (2019a, 9 de julio). Resolución 40590 de 2019. Por la cual se define e implementa un mecanismo que promueva la contratación de largo plazo para proyectos de generación de energía eléctrica complementario a los mecanismos existentes en el Mercado de Energía Mayorista en cumplimiento de los objetivos establecidos en el Decreto 0570 de 2018. https://www.minene rgia.gov.co/documents/10180//23517//48155-res. +4+0590+del+97-20192019-07-09-120048+\%281\%29.pdf

MME (2019b, 9 de julio). Resolución 40591 de 2019. Por la cual se convoca a la subasta de contratación de largo plazo para proyectos de generación de energía eléctrica y se definen los parámetros de su aplicación. http://legal.le gis.com.co/document/Index?obra=legcol\&document=legcol_3b67036 c9db54b17a1673ed00579cd06 
Castaño-Gómez y García-Rendón: Análisis de los incentivos económicos en la capacidad...

Moreno, J. (2017). Incentivos tributarios para generación de energía a través de fuentes no convencionales en Colombia. Revista de Derecho Fiscal, 11, 127-149. https://doi.org/10.18601/16926722.n11.07

Navigant Consulting (2007). Arizona solar electric roadmap study. Full Report. https://repository.asu.edu/attachments/76394/content/AZ_solar_elect ric_roadmap.pdf

Palmer, J., Sorda, G. \& Madlener, R. (2015). Modeling the diffusion of residential photovoltaic systems in Italy: An agent-based simulation. Technological Forecasting and Social Change, 99, 106-131. https://doi. org/10.1016/j.techfore.2015.06.011

Pillot, B., Muselli, M., Poggi, P. \& Batista, J. (2019). Historical trends in global energy policy and renewable power system issues in Sub-Saharan Africa: The case of solar PV. Energy Policy, 127, 113-124. https://doi.or g/10.1016/j.enpol.2018.11.049

Rogers, E. (1962). Diffusion of Innovations. New York: The Free Press.

Saldivia, D., Rosales, C., Barraza, R. \& Cornejo, L. (2019). Computational analysis for a multi-effect distillation (MED) plant driven by solar energy in Chile. Renewable Energy, 132, 206-220. https://doi.org/10.1016/j.re nene.2018.07.139

Simsek, Y., Lorca, Á., Urmee, T., Bahri, P. A. \& Escobar, R. (2019). Review and assessment of energy policy developments in Chile. Energy Policy, 127, 87-101. https://doi.org/10.1016/j.enpol.2018.11.058

Smith, A. (1794). Investigación de la naturaleza y causas de la riqueza de las naciones (Tomo I). Vallalodid: oficina de la viuda é hijos de Santander.

UPME (Unidad de Planeación Minero-Energética) (2015a). Integración de las energías renovables no convencionales en Colombia. http://wwwl.upme.go v.co/DemandaEnergetica/INTEGRACION_ENERGIAS_RENOV ANLES_WEB.pdf

UPME (2015b, 5 de junio). Resolución 281 de 2015. Por la cual se define el límite máximo de potencia de la autogeneración a pequeña escala. http: //extwprlegs1.fao.org/docs/pdf/col146970.pdf 
UPME (2017). Plan de expansión de referencia generación - transmisión 2017 2030. https://www1.upme.gov.co/Energia_electrica/Plan_GT_2017_2 031_PREL.pdf

UPME (2018a). Informe de registro proyectos de generación. http://www.siel.g ov.co/Generacion_sz/Inscripcion/2018/Registro_Sept_2018.pdf

UPME (2018b). Informe mensual-solicitud de certificación de proyectos de Fuentes No Convencionales de Energía (Fnce)-Incentivos Ley 1715 de 2014. https://www1.upme.gov.co/Documents/Energias_renovables/Incentiv os_Ley_1715/InformeNo12_FNCE.pdf

Washburn, C. \& Pablo-Romero, M. (2019). Measures to promote renewable energies for electricity generation in Latin American countries. Energy Policy, 128, 212-222. https://doi.org/10.1016/J.ENPOL.2018.12.059

Winter, S. \& Schlesewsky, L. (2019). The German feed-in tariff revisited - an empirical investigation on its distributional effects. Energy Policy, 132, 344-356. https://doi.org/10.1016/j.enpol.2019.05.043

Zhao, J., Mazhar, E, Celik, N. \& Son, Y. (2011). Hybrid agent-based simulation for policy evaluation of solar power generation systems. Simulation Modelling Practice and Theory, 19(10), 2189-2205. https: //doi.org/10.1016/j.simpat.2011.07.005

Zhao, Z., Chen, Y. \& Chang, R. (2016). How to stimulate renewable energy power generation effectively? - China's incentive approaches and lessons. Renewable Energy, 92, 147-156. https://doi.org/10.1016/j.renene.2016. 02.001 